Ann. Zootech., I962, 11 (I), 5-38.

\title{
PRODUCTION LAITIERE DES BREBIS PRÉALPES DU SUD PENDANT LES PHASES D'ALLAITEMENT, DE SEVRAGE ET DE TRAITE
}

\author{
G. RICORDEAU, R. DENAMUR \\ avec la collaboration technique de P. Petrequin \\ Station de Recherches sur l'Élevage, Station de Recherches de Physiologie animale, \\ Centre national de Recherches zootechniques, Jouy-en-Josas (Seine-et-Oise).
}

SOMMAIRE

La production laitière des brebis Préalpes a été étudiée au cours des phases successives d'allaitement, de sevrage et de traite (I6 lots et 250 lactations).

I. - La connaissance de la production laitière des Brebis traites quelques jours après la mise bas a permis de déterminer la lactation de référence. Celle-ci se caractérise par une phase croissante (5० premiers jours de lactation au maximum) suivie d'une phase décroissante, l'ensemble couvrant une période d'environ 175 jours.

Le maximum est peu élevé ( 1,25 litre) et se situe en moyenne à 25 jours (tableau 3 ). Il est d'autant plus précoce que son niveau est plus élevé.

D) $5_{5}{ }^{\mathrm{e}}$ au $\mathrm{I}_{5}{ }^{\mathrm{e}}$ jour de lactation, le taux de décroissance hebdomadaire de la production laitière est de 7 p. Ioo (tableau 4).

Ia production laitière obtenue au cours de chacun des 2 premiers mois de lactation représente $25 \mathrm{p}$. 100 de la production totale obtenue en I 75 jours de traite.

II. - La phase croissante de la lactation des brebis en période d'allaitement a été étudiée chaque semaine, par pesée des agneaux avant et après la tétée.

Pour les brebis avec I et 2 agneaux, en moyenne, le maximum se situe respectivement à 19 et 16 jours et atteint $\mathrm{I}, 7$ et $2,3 \mathrm{~kg}$ (soit une différence de $34 \mathrm{p}$. Ioo-tableau 3 ).

III. - Quel que soit le mode de sevrage (brutal ou progressif - tableau 8), le nombre d'agneaux élevés (tableau 9), la durée de la période d'allaitement ( 15,30 ou 45 jours - tableau Io), la production de lait obtenue à la traite, après la période d'allaitement est (à l'exception des 2 premières semaines) pour la même période de lactation, tout à fait comparable en quantité et persistance à la production des brebis soumises à la traite dès la mise bas.

IV. - La comparaison des performances enregistrées à la traite et à la tétée entre la fin d'allaitement et le début de la traite dans le cas d'un sevrage brutal, entre périodes successives de traite et de tétée dans le cas d'un sevrage progressif, montre que la traite ne permet d'obtenir que 60 à 80 p. Ioo du lait normalement tété par l'agneau (tableau i I). La différence [lait tété - lait trait] est en corrélation positive élevée avec la ${ }_{1}{ }^{\text {re }}$ de ces variables et en corrélation légèrement négative avec la seconde ; elle dépend du poids des agneaux, sauf lorsque ceux-ci sont suffisamment vigoureux pour consommer tout le lait que leur mère est capable de produire.

Il existe effectivement une relation positive entre les quantités de lait obtenues à la traite et celles consommées par les agneaux, mais ces dernières n'ont qu'une valeur relative et ne peuvent être intégrées sans correction dans le calcul de la lactation totale.

Pour être efficace, il apparaît nettement que la sélection des brebis exploitées pour leur production laitière doit reposer essentiellement sur les performances obtenues à la traite. 
V. - I'adoption du sevrage progressif ne supprine pas la rétention du lait consécutive au sevrage complet des agneaux mais permet de commencer la traite un peu plus tôt (le lait de la traite partielle étant cependant plus pauvre en matière grasse — tableau I 3) et surtout de diminuer les risques de mammites notamment chez les brebis ayant allaité 2 agneaux.

Immédiatement après la traite en cours de sevrage progressif, l'agneau est capable de récupérer une quantité de lait représentant 3 I p. roo du volume de lait trait ; en remplaçant la tétée de l'agneau par une traite effectuée après injection de I I d'ocytocine, on obtient 62 p. Ioo de ce même volume (tableau I 5).

La présence de l'agneau, lors des traites réalisées en cours de sevrage né permet pas d'obtenir une meilleure vidange de la mamelle (tableau I4).

\section{INTRODUC'TION ET BIBLIOGRAPHIE}

Dans quelques régions d'élevage intensif (en Allemagne notamment avec les brebis Milchschaf) les brebis sont séparées de leurs agneaux dès la mise bas et soumises au même régime de traite que les vaches laitières. Au contraire, dans toutes les régions méditerranéennes, productrices de lait de brebis, la lactation comprend traditionnellement 3 périodes successives :

- la période d'allaitement;

- une période intermédiaire correspondant au sevrage des agneaux ;

- la période de traite.

Ces trois phases ont des durées et des modalités différentes suivant la part réservée à la production du lait et à la production d'agneaux. Elles ont fait l'objet d'un certain nombre d'études dont voici l'essentiel.

\section{I. - Période d'allaitement}

La production laitière des brebis pendant cette période a été estimée par de nombreux chercheurs, sur plusieurs races (exploitées ou non exploitées pour leur production laitière) et à l'aide de techniques assez voisines (cf. RICORDEAU, BOCCARD, Denamur, I960). De l'ensemble des résultats, il apparaît que le maximum de production journalière est atteint au cours des 4 premières semaines de lactation. Le niveau de production, l'âge et l'importance de l'agnelée influencent cependant la courbe de lactation.

\section{Niveau de production:}

Bonsma (I939 et I944), Walrace (1948), Barnicoa't, Logan, Grant (I949) et STARKE (I953) constatent que pour des brebis de même âge, les faibles productrices allaitant I agneau, atteignent leur maximum dès les premiers jours post-partum, alors que la production laitière des fortes productrices augmente en moyenne jusqu'à 1a $4^{\mathrm{e}}$ semaine de lactation. STARKE (I953) et Bonsma (I944) à partir de croisements entre races médiocres laitières (Mérinos et Blackhead Persian) et races meilleures laitières (Romney, Border Leicester ou Lorset horn) arrivent même à la conclusion suivante : l'absence d'un maximum de production journalière est une caractéristique des races non améliorées. Cependant, Hunter (1956) n'observe pas de différence dans le stade d'apparition du maximum de production en fonction du niveau de production (brebis Border Leicester et Welsh Mountain). 
Age :

Les résultats obtenus sont très fragmentaires et ne permettent pas d'observer l'allure moyenne des lactations pour des brebis de même production, mais d'âges différents. Ainsi Barnicoats et al. (I949) constatent que des brebis Romney de 6 ans ont une production laitière supérieure de $15 \mathrm{p}$. Ioo à celle des brebis de 2 ans. Ces 2 catégories de brebis présentent des courbes de lactation différentes : la production laitière des brebis adultes est croissante de la $\mathrm{I}^{\mathrm{re}}$ à la $^{\mathrm{e}}$ semaine, alors que pour les jeunes brebis, le maximum atteint dès la $I^{\text {re }}$ semaine se maintient au cours des 4 semaines suivantes.

Importance de l'agnelée:

Les bessonnières ont en moyenne une production laitière supérieure de $37 \mathrm{p}$. Ioo à celle des brebis allaitant des simples (tableau $\mathrm{I}$ ). Leur production maximum journalière est nettement plus élevée et se situe dans l'ensemble plus précocement : Barnicoat et al., (I956), Hunter (I956), Davies (1958), GuYer et Dyer (I954), WALLACE (I948). Les contrôles effectués par ces derniers auteurs sur des brebis allaitant des triplés indiquent un maximum très élevé, mais aussi une décroissance très rapide de la production laitière dès la $2^{\mathrm{e}}$ semaine de lactation.

TABLEAU I

Période d'allaitement. Production des bessonnières en p. Ioo de la production des brebis allaitant un agneau

\begin{tabular}{|c|c|c|}
\hline & p. 100 & $\begin{array}{l}\text { Semaines } \\
\text { de lactation }\end{array}$ \\
\hline 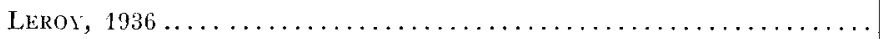 & 127 & \\
\hline 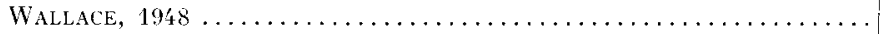 & 150 & $1-16$ \\
\hline Barnicoat, Logan et Grant, $1949 \ldots \ldots \ldots \ldots \ldots \ldots$ & 135 & $1-12$ \\
\hline 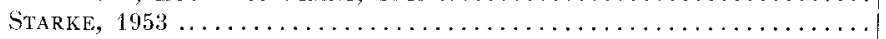 & 150 & $1-13$ \\
\hline 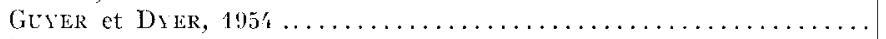 & 130 & $1-9$ \\
\hline 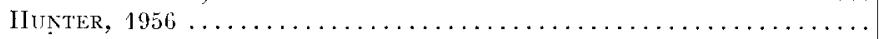 & 136 & $1-16$ \\
\hline 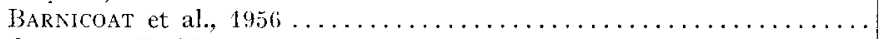 & $13_{t}^{\prime}$ & $3-9$ \\
\hline 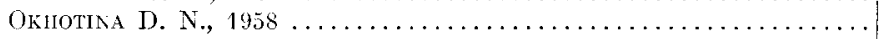 & $1 \geq 8$ & $1-18$ \\
\hline 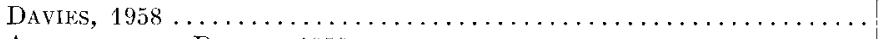 & 151 & $1-10$ \\
\hline 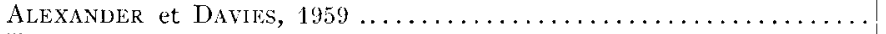 & 118 & $2-8$ \\
\hline 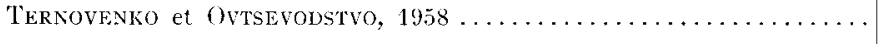 & 110 & $1-11$ \\
\hline Moyenne & 136,6 & \\
\hline Ricordenu, Denamur (Préalpes) : & & \\
\hline $1956(n=13) \ldots \ldots \ldots \ldots \ldots$ & 136,3 & \\
\hline 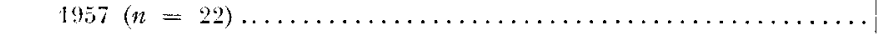 & $138, \ddot{2}$ & $1-6$ \\
\hline 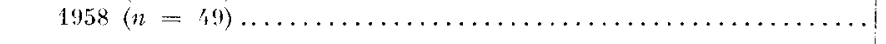 & 135,0 & \\
\hline Moyenne ....... & 137,2 & \\
\hline
\end{tabular}

Ainsi l'agnelée a une influence dominante sur l'allure des courbes de lactation et WALLACE (I948) conclut logiquement que le maximum de production journalière dépend plus de l'appétit des agneaux que de l'aptitude laitière réelle de leur mère. En outre, les agneaux des brebis adultes n'allaitant qu'un agneau (ou des meilleures laitières) ne consomment pas tout le lait produit, ce qui conduit à sousestimer la production laitière des mères au cours de la phase initiale d'allaitement. Suivant BARnicoat et al., cette sous-estimation est, en 3 semaines de Io et 40 p. xoo respectivement pour les brebis de 2 et 6 ans. 


\section{2. - Période de sevrage}

La plupart des études relatives à la détermination de la quantité de lait consommé par les agneaux concernent des brebis non soumises à la traite. Aussi, l'influence du sevrage des agneaux sur la production laitière de leur mère, n'a-t-elle fait l'objet que d'un nombre restreint d'observations. Cependant, elles s'accordent toutes pour constater que le passage de l'allaitement à la traite se traduit par une chute de production. FINCI (I957) estime par exemple que l'utilisation du premier contrôle laitier en vue de l'évaluation de la production laitière obtenue à l'allaitement antérieurement à ce contrôle, sous-estime de $32 \mathrm{p}$. Ioo la production de lait réellement consommée par les agneaux. Pour GEORgIOU (1960) la différence observée entre les I5 derniers jours d'allaitement et les I5 premiers jours de traite (sevrage à 60 jours) n'est que de II p. roo pour les brebis avec I agneau, contre $22 \mathrm{p}$. Ioo pour les bessonnières. Les résultats de cet auteur ne sont cependant pas parfaitement concluants, car les bessonnières de race Chios et Mytilènes citées par GEORGIOU ont des performances laitières pendant l'allaitement inférieures à celles des brebis avec simples.

En ce qui concerne les traites effectuées en période d'allaitement, ConstantiNESCU et Gondos ( $\left.195^{8}\right)$ montrent que les agneaux doivent toujours être présents à la traite ou téter une moitié de la mamelle, sinon, on ne retire que 60 à $75 \mathrm{p}$. Ioo du lait et 40 à $50 \mathrm{p}$. Ioo de la matière grasse dans la moitié traite. Cror.cA et al. (Ig60) constatent également que la tétée des agneaux après une période de 24 heures de traite permet de récupérer $38 \mathrm{p}$. Ioo de la production totale journalière.

Si on compare les performances laitières obtenues à la traite et à la tétée, non plus sur des périodes successives de lactation, mais pour une même phase, on constate aussi que la tétée est plus efficace que la traite. Le la confrontation des productions laitières de 2 groupes de Io brebis Leine, les premières traites manuellement depuis la mise bas, les secondes allaitant leur agneaux, Scheinacker (I927) conclut que la production des brebis traites est inférieure de $40 \mathrm{p}$. Ioo à celles des brebis allaitantes, ces dernières ayant cependant une plus courte durée de lactation.

\section{3. - Période de traite}

Cette période commence dès la mise bas pour les brebis de Frise orientale ou après les périodes d'allaitement et de sevrage pour les brebis des régions méditérranéennes.

In ce qui concerne les brebis Milschaf, nous disposons de nombreux résultats sur les productions laitières totales (ScheINGrabER, I934; CREMER, I935; EBBINGHAUS, I949; Buitkamp, I952). Par contre, les références concernant les courbes de lactation n'existent pas à notre connaissance. Il est paradoxal de constater que les données que nous possédons sur la variation de la production laitière avec le stade de lactation concernent les brebis Awasi et Tsigä̈: données obtenues dans des conditions particulières, puisque FincI (I957) retient uniquement les lactations de brebis n'ayant pas allaité leur agneau pendant plus de I5 jours, alors qu'OGNJANovic (I958) se réfère à des traites répétées chaque quinzaine au cours des 2 ou 3 premiers mois d'allaitement. Les résultats de ces deux auteurs sont rapportés dans le tableau 2 .

Pour les brebis méditerranéennes, le mode de sevrage, l'âge des brebis et le nombre d'agneaux allaités, sont susceptibles d'avoir des répercussions sur la production laitière à la traite. Certains auteurs ont essayé de déterminer l'influence de ces différents facteurs; 
- mode de sevrage : les résultats sont peu significatifs par suite de l'insuffisance des données (KIRSCH, I943, I 7 brebis en 3 lots) et d'un sevrage trop tardif (Pospeiov, I940, sevrage à I5o jours).

— importance de l'agnelée : les travaux de Mason et DASSat, (I953) Al faXander et DAviEs (I959) font apparaitre nettement que les performances supérieures des bessonnières ne se manifestent que pendant la période d'allaitement, lorsque la brebis élève effectivement ses 2 agneaux.

- âge : parmi les nombreuses études réalisées sur l'augmentation de la production laitière avec l'âge, celles de CREMER (I935), BetTini (I952), Mason et Dassat (I953), Bonelil (I955), Finci (I957), MEREU (I957), OGnjanovic (I958) s'accordent à situer le maximum de production par lactation au niveau de la $3^{\mathrm{e}}$ lactation; 1 'accroissement de production enregistré au cours des lactations suivantes étant, soit négligeable, soit discutable par suite de l'élimination d'un grand nombre de brebis. En fait, les performances enregistrées ne sont pas toutes comparables, les unes concernant la traite sur toute la lactation, les autres tenant compte de l'estimation de production laitière initiale correspondant à l'allaitement (estimation basée sur le résultat du premier contrôle laitier ou des traites effectuées après tétée des agneaux).

Comme nous pouvons le constater à la suite de ce bref rappel bibliographique, la production laitière des brebis en période d'allaitement a fait l'objet de nombreuses investigations mais les performances des brebis soumises à la traite n'ont pas été étudiées systématiquement. Les résultats obtenus dans des conditions parfois très différentes et sans faire la distinction entre les périodes d'allaitement, de sevrage et de traite, ne sont pas suffisamment comparables. Il nous a paru important d'étudier la production laitière au cours de chacune de ces trois périodes en vue :

Io de normaliser le contrôle des performances et définir les paramètres de production laitière permettant au généticien d'entreprendre une sélection plus efficace.

$2^{\circ}$ de fournir au physiologiste des observations lui permettant de poser correctement les problèmes de la physiologie de la traite des brebis.

\section{ÉTUDE EXPÉRIMENTALE}

Afin de déterminer la production laitière des brebis Préalpes au cours des phases successives d'allaitement, de sevrage et de traite, ainsi que les relations existant entre ces performances, nous avons étudié la production laitière des brebis dans les conditions suivantes :

$\mathrm{I}^{0}$ Allaitement depuis la parturition;

$2^{\circ}$ Traite dès la parturition;

$3^{\circ}$ Allaitement de durée constante, suivi de la traite, après différents modes de sevrage (sevrage brutal ou progressif);

$4^{\circ}$ Allaitement de durées variables (2I, 30 et 45 jours) suivi de la traite après une même technique de sevrage.

Nous ne nous étendrons pas sur les résultats relatifs au point I, c'est-à-dire au 


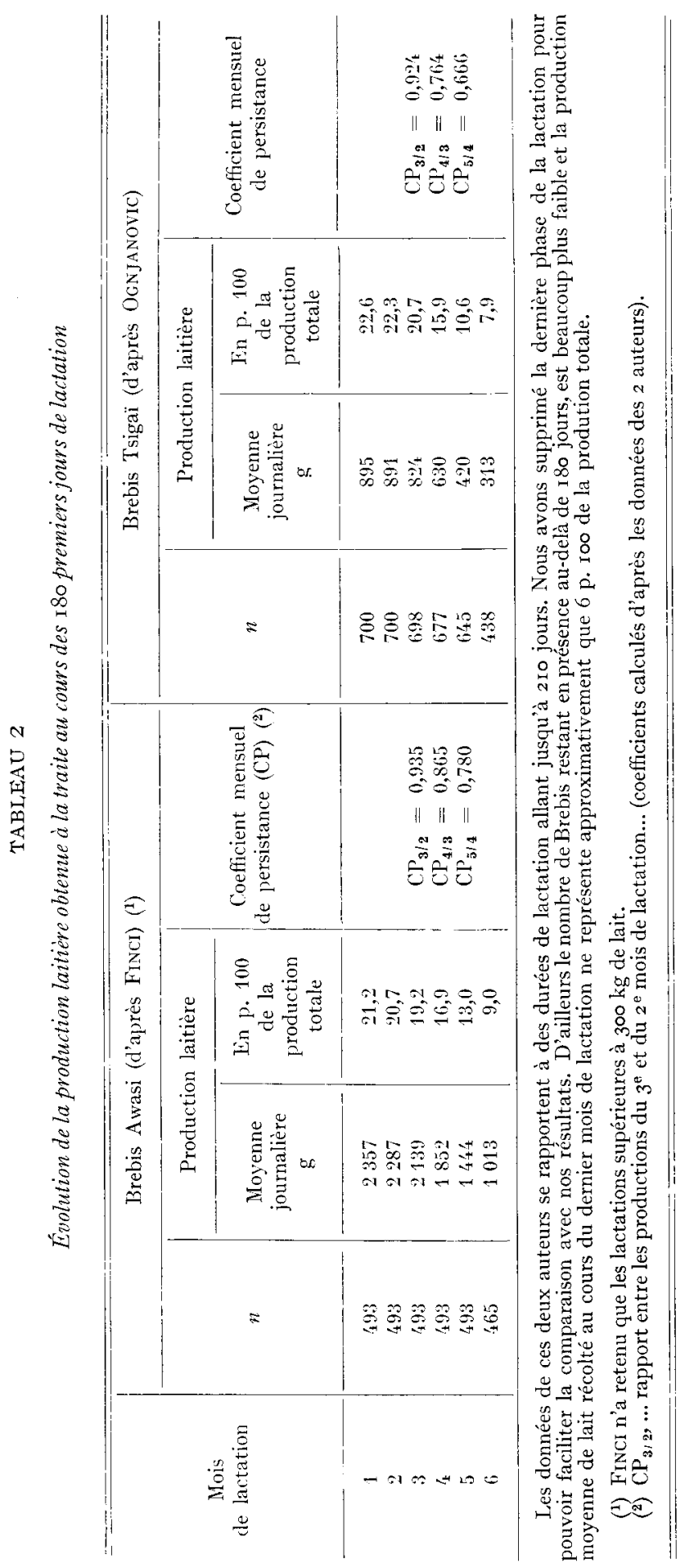


contrôle de la quantité de lait consommé par les agneaux pendant une longue période de la lactation. De tels contrôles sont difficiles à réaliser et les performances qu'ils permettent d'obtenir sont peu représentatifs. En effet, après quelques semaines d'allaitement les agneaux acquièrent leur indépendance alimentaire et les mères se dérobent. Les quelques courbes de lactation obtenues dans ces conditions et rapportées à la fin de cette étude (graphique 7) se caractérisent toutes par une faible persistance.

On peut évidemment envisager le maintien de la sécrétion grâce à des adoptions successives comme cela a été fait avec succès chez la ratte (BRUCE, I96I). Une telle technique ne manque pas d'intérêt, mais risque de présenter de sérieuses difficultés pratiques et de ne pas être suffisamment simple et répétable pour servir de référence.

Les brebis soumises à la traite dès la mise bas ont des productions laitières qui ne sont pas perturbées par la présence des agneaux, ou le régime de sevrage. La lactation obtenue dans ces conditions peut donc être choisie comme référence.

\section{A. - MATÉRIEI, E'T MÉTHODE}

$$
\text { I. - Matériel }
$$

Les brebis laitières de race Préalpes, utilisées pour nos expériences, ont été logées dans 2 bergeries du Centre national de Recherches zootechniques (l'une située à Jouy-en-Josas, l'autre à la ferme de Brouessy) et réparties en i 6 lots. Ces lots dont les caractéristiques sont données dans les différents tableaux, se distinguent par les conditions de sevrage et de traite ainsi que par le nombre d'agneaux à la naissance.

Deux méthodes de sevrage ont été appliquées : le sevrage brutal et le sevrage progressif. Dans le premier cas, on passe de l'allaitement à la traite sans transition. Dans le second cas, les brebis allaitent leurs agneaux pendant la nuit (de $\mathrm{I} 8 \mathrm{~h}$ à $6 \mathrm{~h}$ ) et sont traites une seule fois par jour (à I $8 \mathrm{~h}$ ) pendant les I 4 jours précédant le sevrage complet. Suivant les lots, le sevrage progressif a commencé après I 4,2 I ou 42 jours d'allaitement complet; il a toujours duré 2 semaines.

Presque toutes les brebis ont été maintcnues en bergerie depuis l'agnelage jusqu'à la fin de la lactation. Elles ont été soumises en moyenne, au même régime alimentaire, les bonnes brebis disséminées dans les différents lots n'ayant reçu aucune supplémentation en fonction de leur niveau de production.

I.e taux de féconditié moyen observé a été de 145 agneaux pour 100 mères ayant mis bas.

250 lactations ont été analysées.

\section{II. - Méthodes d'obtention de la production laitière (1)}

\section{Pendant l'allaitement :}

L'estimation de la quantité de lait consommée par les agneaux a été obtenue par pesée des agneaux avant et après la tétée suivant une méthode précédemment décrite : pesées toutes les $2 \mathrm{cu}$ 3 heures sur une demi-journée (RICORDEAU, Boccard, Denamur, I960).

\section{Pendant la traite :}

La traite est pratiquée deux fois par jour, soit à la main, soit à la machine (les comparaisons effectuées en I957 et I958 entre ces deux modes de traite n'ont révélé aucune différence).

Dans les 2 bergeries, pendant le sevrage progressif, comme après le sevrage définitif, la traite mécanique (Machine à traire Alfa Laval à Brouessy, Gascoigne à Jouy-en-Josas) est suivie d'un égouttage machine, puis d'un égouttage manuel appelé "repasse ». Les quantités de lait récoltées lors de la traite mécanique et de la repasse ont été enregistrées quotidiennement.

Pendant le sevrage progressif:

Pour mesurer la quantité de lait consommée par les agneaux en i 2 heures on effectue 7 pesées à 2 heures d'intervalle, la première, se plaçant immédiatement après la fin de la « repasse manuelle ".

(I) Dans l'analyse des performances, nous n'avons pas abordé l'aspect sanitaire qui a fait l'obiet d'une étude spéciale (Plommet, Ricordeat, I960). 


\section{III. - Méthodes analytiques}

En 1958, 5 lots ont été suivis du point de vue richesse en matière grasse (Gerber) : analyses hebdomadaires des échantillons pondérés des 2 traites quotidiennes.

En 1959, les laits de mélange du sevrage progressif ont été analysés en vue d'obtenir la teneur en matière grasse (gerber), la teneur en matières azotées totales (Kjeldahl) et le temps de coagulation (présure 2 p. 100), à part ir de prélèvement quotidiens.

\section{B. - Résultats}

\section{I. - Traite dès la fin de la phase colostrale -- Lactation de vétérence}

Cette étude concerne I 26 lactations enregistrées depuis 1956 à partir de 8 lots. Sur ces lactations, 27 seulement ont une durée supérieure à 150 jours.

Pour toutes ces lactations, la traite commence au plus tard après 5 oul 6 jours d'allaitement. Le fait de ne pas avoir soustrait les agneaux de leur mère dès la naissance (ou d'avoir maintenu constamment les brebis dans la même bergerie, en présence d'autres brebis allaitant leurs agneaux) est une source possible de variation. Une étude est actuellemınt en cours pour élucider ce problème, mais il semble bien d'après les premiers résultats obtenus, que la séparation des agneaux à la mise bas et 1'isolement des mères, ne modifient pratiquement pas l'adaptation des brebis à la machine à traire.

\section{$\mathbf{I}^{\mathbf{o}}$ Phase croissante}

La production laitière totale au cours de la phase initiale de la lactation résulte essentiellement de 2 paramètres :

-- le niveau correspondant au maximum de production ;

- le stade auquel est atteint ce maximum.

Pour atténuer les variations journalières, la production maximum correspondant à chaque lactation a été calculée sur 2 jours consécutifs de contrôle. Les résultats obtenus pour les 2 catégories de brebis (avec I et 2 agneaux à la mise bas) ont été classées par numéro de lactation ( $\mathrm{I}^{\mathrm{er}}, 2^{\mathrm{e}}, 3^{\mathrm{e}}$ et plus). Fn ce qui concerne les bessonnières, nous avons négligé les performances des premières traites, pour ne retenir que le maximum intervenant normalement après la phase d'adaptation à la traite.

Production maximum journalière ('Tableau $3 a$ ). - La production maximum est en moyenne de I,25 litre pour l'ens smble des données ; elle augmente légèrement avec l'âge des brebis et est plus élevée chez les bessonnières mais uniquement pour les lactations adultes ( $r, 55$ contre $I, 30$ litre).

Stade auquel est atteint le maximum. - Ce stade se situe en moyenne à 25 jours ; il apparaît plus précocement pour les brebis adultes et pour les bessonnières. C'est ainsi que dans la catégorie de brebis avec I agneau, le pourcentage d'animaux ayant atteint leur maximum à 20 jours est de $2 \mathrm{I}$ en $\mathrm{I}$ er, 33 en $2^{\mathrm{e}}$ et 43 en lactation adulte.

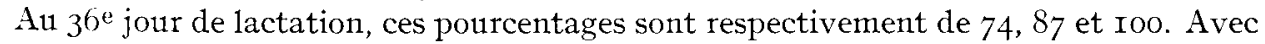
les bessonnières, ces valeurs sont légèrement supérieures. Pour la majorité des brebis, la phase croissante est donc déjà terminée dès le début du $2^{\mathbf{e}}$ mois de lactation ; elle dépasse rarement le $5 \mathrm{o}^{\mathrm{e}}$ jour pour la totalité des brebis.

Relation entre la production maximum et le stade auquel elle intervient. - I1 existe une corrélation négative entre ces 2 variables $(r=-0,38$ pour les 86 brebis avec simples et $r=-0,39$ pour les 40 bessonnières). En effet, pour les brebis excellentes 
PRODUCTION LAITIÈRE DES BREBIS

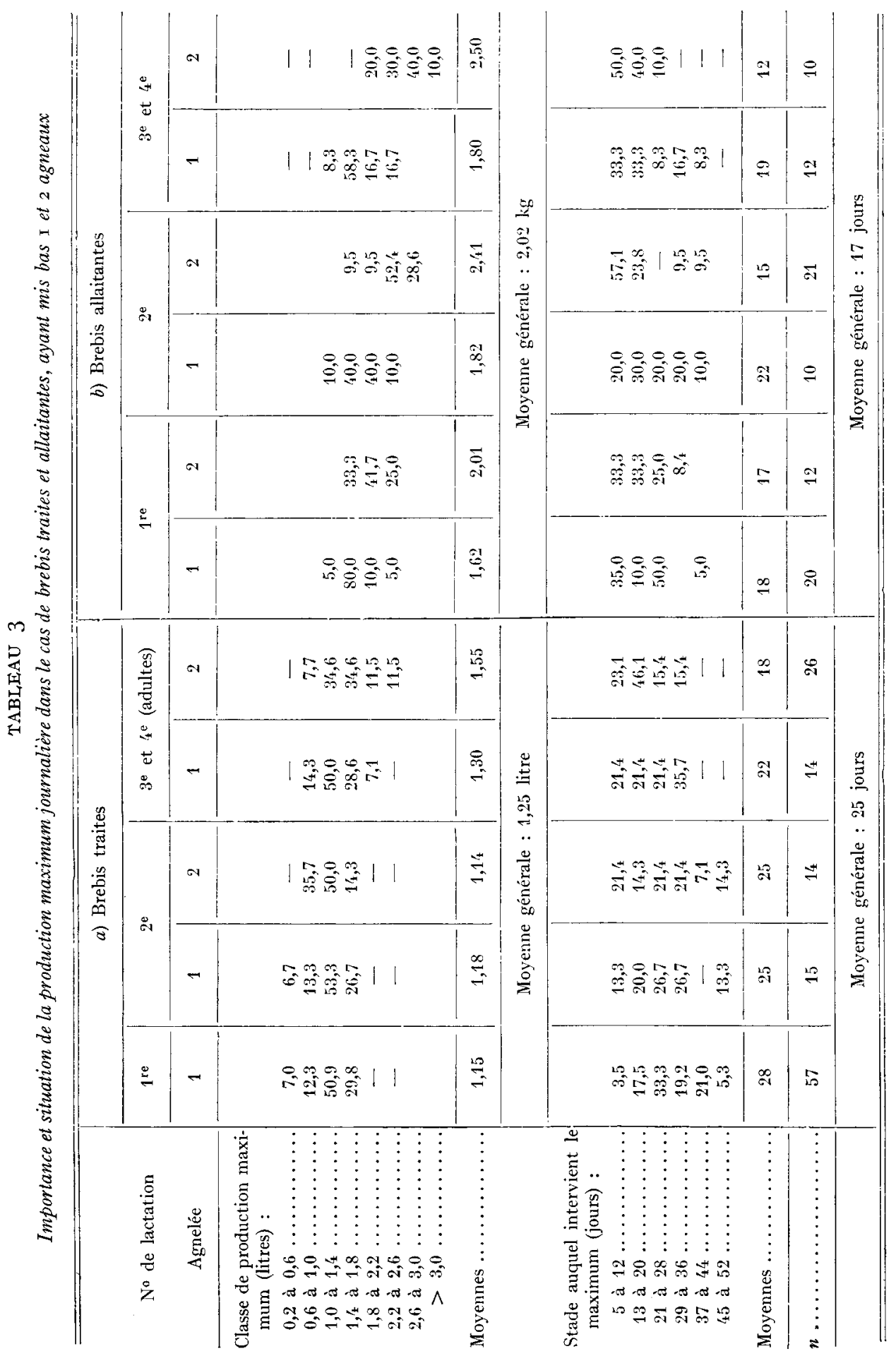


laitières, le maximum est très prononcé et se manifeste très tôt (entre 6 et 20 jours), alors qu'il n'apparaît pas avant 40 jours pour les médiocres laitières. Après $5^{n}$ jours, toutes les courbes de lactation tendent à se rapprocher (graphique I).

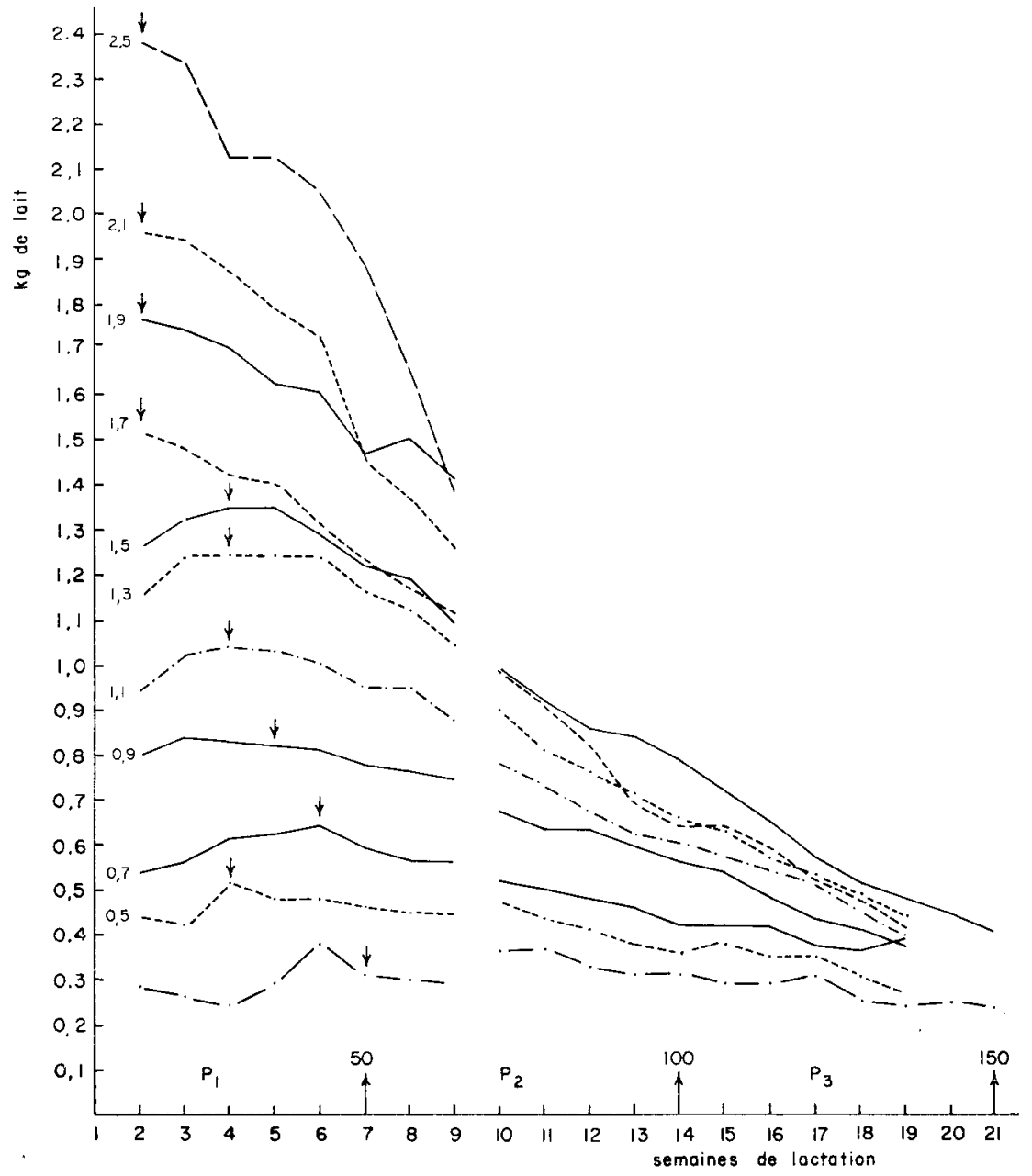

GRAPHIQUe I. - Evolution de la production laitière au cours des I 50 premiers jours de lactation en fonction de la production maximum iournalière

60 premiers jours de lactation (I 28 brebis);

90 jours suivants ( 99 brebis);

$\downarrow$ Situation du maximum moyen journalier calculé sur 2 jours consécutifs.

Ces résultats rendent difficiles la recherche d'une expression mathématique de la phase initiale de la lactation et expliquent pourquoi l'utilisation du premier contrôle laitier vers 35 jours, en vue de l'estimation de la production laitière antérieure, conduit à sous-estimer les performances des meilleures laitières.

\section{$2^{\circ}$ Phase décroissante.}

Les 50 premiers jours de lactation (période I) couvrent, comme nous venons de le voir la totalité de la phase d'accroissement de la sécrétion laitière. 
Du $5^{\mathrm{e}}$ au $\mathrm{IOO}^{\mathrm{e}}$ (période 2 ) et du $\mathrm{IOO}^{\mathrm{e}}$ au $\mathrm{I}_{5} \mathrm{O}^{\mathrm{e}}$ jour de lactation (période 3 ) on peut admettre valablement une décroissance linéaire (graphique I). I'ailleurs, les coefficients hebdomadaires de persistance diminuent légèrement avec le stade de la lactation $(0,937$ et 0,920 pour les périodes 2 et 3 respectivement, tableau 4$)$.

\section{TABLEAU 4}

Évolution de la production laitière des brebis "Préalpes du sud" soumises à la traite une semaine après la mise bas

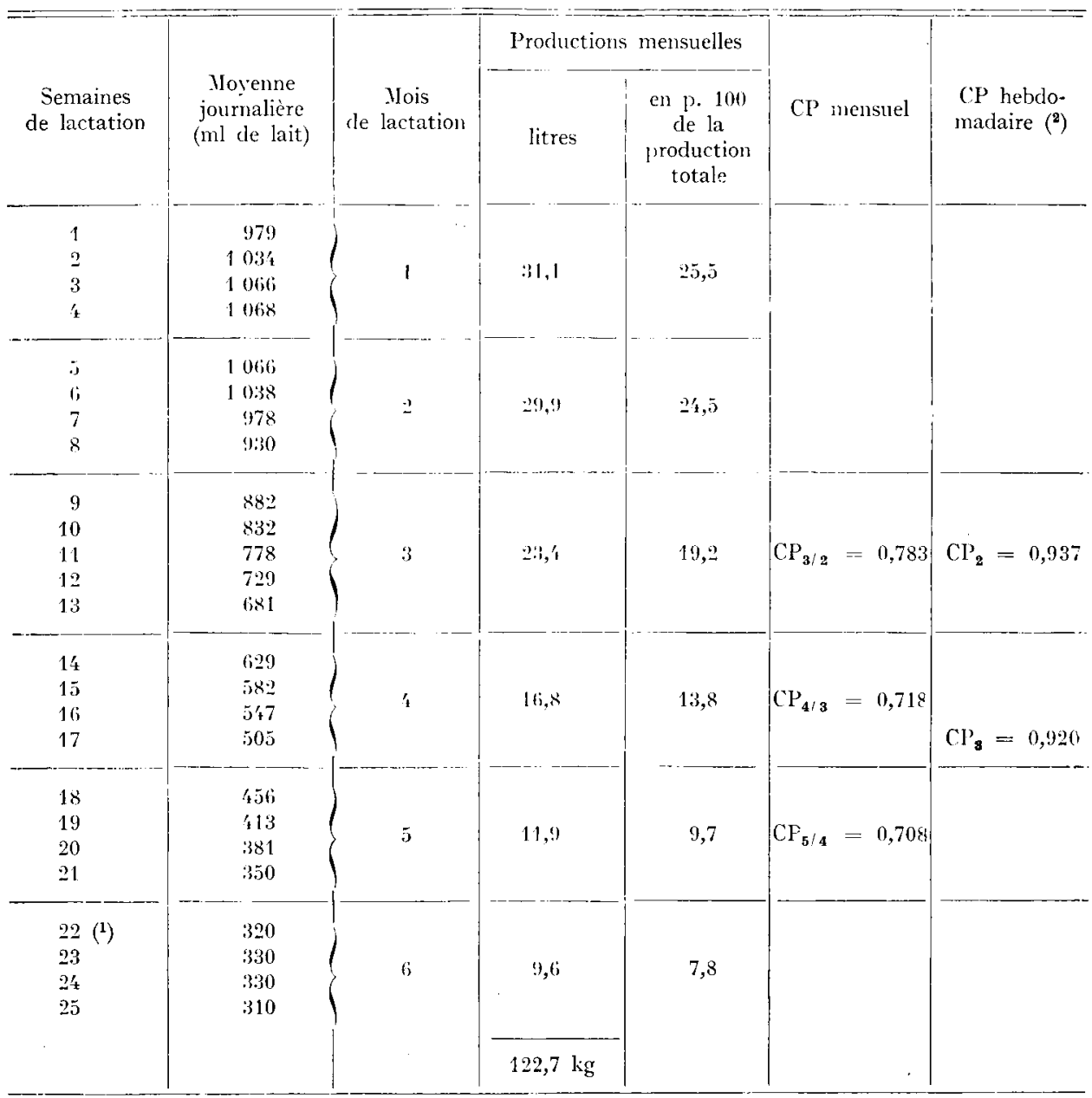

(1) Estimation sur 80 brebis.

(2) Le coefficient de persistance hebdomadaire est le rapport entre la production de la semaine d'ordre $(n+1)$ à celle de la semaine d'ordre $n . \mathrm{CP}_{2}$ et $\mathrm{CP}_{3}$ sont les moyennes des CP hebdomadaires pour les périodes $2\left(50^{\mathrm{e}}\right.$ au $100^{\mathrm{e}}$ jour de lactation) et $3\left(100^{\mathrm{e}}\right.$ au $\left.150^{\mathrm{e}} \mathrm{j}\right)$.

Si l'on considère les contrôles mensuels, les coefficients $\mathrm{CP} \mathrm{3}_{\mathbf{3}}, \mathrm{CP}_{4 / 3}$, et $\mathrm{CP}_{5 / 4}$ (rapports entre les productions de 2 mois consécutifs) sont respectivement de 78,72 
et $7 \mathrm{r}$ p. IOO : valeurs comparables à celles des brebis Tsigaï (OGNJ ANovic), mais nettement inférieures à celles des Awasi (FINCI) tableaux I et 4.

$3^{\circ}$ Relations entre les performances obtenues au cours des différentes phases de la lactation de référence.

En calculant la production laitière totale obtenue à la traite dans les I75 premiers jours de lactation, on constate (comme pour les brebis Awasi et Tsigai) que les brebis Préalpes arrivent à produire en moyenne $25 \mathrm{p}$. Ioo de leur production au cours de chacun des 2 premiers mois de lactation (tableau 4).

En fait, au-delà du $\mathrm{I}_{5} \mathrm{O}^{\mathrm{e}}$ jour de lactation la production laitière obtenue est relativement très faible. On peut donc négliger la "queue " de la lactation et calculer les relations existantes entre les productions respectives des 3 périodes de 50 jours $\left(\mathrm{P}_{1}, \mathrm{P}_{2}, \mathrm{P}_{3}\right)$, la production totale des $\mathrm{I}_{50}$ jours $\left(\mathrm{P}_{t}\right)$, la production maximum journalière $\left(\mathrm{M}_{j}\right)$, la chute de production de la $2^{\mathrm{e}}$ à la $3^{\mathrm{e}}$ période $\left(\mathrm{P}_{2-3}\right)$ et le coefficient de persistance hebdomadaire correspondant à l'ensemble des périodes 2 et $3\left(\mathrm{CP}_{2+3}\right)$. Les résultats obtenus sont inscrits dans le tableau 5 .

\section{TABLEAU 5}

Corrélations entre les performances laitières obtenues à la traite $\dot{a}$ différentes périodes de la lactation.

\begin{tabular}{|c|c|c|c|c|c|c|c|c|}
\hline $\mathrm{l}_{1}$ & $\mathrm{P}_{2}$ & $\mathrm{I}_{3}$ & $\mathrm{I}_{2+3}$ & $l^{\prime} t$ & $\mathrm{P}_{2-3}$ & $\mathrm{CP}_{\mathbf{2}+\mathbf{3}}$ & $\mathrm{P}_{2.3}$ & Production laitière \\
\hline \multirow[t]{2}{*}{$0,93^{* *}$} & & & 0,$6 ; 4^{* *}$ & $0,8: 2 * *$ & $0,75^{* *}$ & $-0,32^{2}$ & & $\mathrm{Mj}$ : maximum journalière \\
\hline & $0,83^{* *}$ & $0,46 * *$ & $0,72 * *$ & $0,91^{* *}$ & $0,78^{* *}$ & $\cdots-0,31^{2}$ & & $\mathrm{P}_{\mathbf{1}}:$ du $1^{\mathrm{er}}$ au $50^{\mathrm{e}} \mathrm{j}$ de lactation \\
\hline & & $0,7 x^{* *}$ & & & & & & $\mathrm{P}_{2} \quad$ : du $50^{\mathrm{e}}$ au $100^{\mathrm{e}} \mathrm{j}$ de lactation \\
\hline & & & & $0,94 * *$ & $0,43^{* *}$ & $0,2,6^{1}$ & $-0,02$ & $\mathrm{P}_{2+3}:$ du $50^{\mathrm{e}} 150^{\mathrm{e}}$ au $\mathrm{j}$ de lactation \\
\hline & & & & & $0,6,3 * *$ & 0,01 & 0,05 & : du $1^{\text {er }}$ au $150^{\mathrm{e}} \mathrm{j}$ de lactation \\
\hline
\end{tabular}

$(* *)$ : Significatif au seuil $\mathrm{P}=0, \mathrm{or}$.

$\mathrm{P}_{3}$ : Production laitière du $100^{\circ}$ au $150^{\mathrm{e}}$ jour de lactation.

$(*)$ : Significatif au seul $\mathrm{P}=0,05$. Différents indices de persistance :

$P_{2-3}=P_{2} \cdots P_{3}$

$\left(\mathrm{P}_{2+3}=\right.$ moyenne des coefficients de persistance hebdomadaires pour les périodes 2 et 3.

$\mathrm{P}_{23}=\mathrm{F}_{2} / \mathrm{P}_{3}$.

Ces résultats concernent 99 lactations de brebis soumises à la traite quelques jours après la mise bas et contrôlées quotidiemnement.

La production laitière initiale estimée d'après $\mathrm{M}_{j}$ et $\mathrm{P}_{1}$ est en liaison étroite avec la production $\mathrm{P}_{2+3}$ correspondant à la phase classique de traite $(r=0,64-0,72)$, mais surtout avec $\mathrm{P}_{t}(r=0,82-0,9 \mathrm{I})$.

I a corrélation entre les performances enregistrées au cours de 2 périodes consécutives, $P_{1}$ et $P_{2}, P_{2}$ et $P_{3}$ est de l'ordre de 0,80 .

La production totale $\mathrm{P}_{\iota}$ est conditionnée plus étroitement par le niveau maximum mum $\mathrm{M}_{j}(r=0,82)$ que par la pente de la lactation $\mathrm{P}_{2-3}(r=0,63)$. Le calcul des corrélations partielles entre $\mathrm{P}_{t}$ et $\mathrm{M}_{j}$ d'une part, $\mathrm{P}_{t}$ et $\mathrm{P}_{2-3}$ d'autre part, le $3^{\mathrm{e}}$ facteur étant maintenu constant, montre que le coefficient de détermination de la production 
totale $\mathrm{P}_{t}$ à partir du niveau maximum $\mathrm{M}_{j}$ est de $46 \mathrm{p}$. Ioo alors que celui correspondant à la régression $\mathrm{P}_{\mathbf{2}-\mathbf{3}}$ est pratiquement nul. Pour améliorer $\mathrm{P}_{t}$, il semble donc beaucoup plus efficace d'améliorer $\mathrm{M}_{j}$ que de chercher à réduire la chute de production laitière en cours de lactation. Cela est d'autant plus valable que le maximum de production est un paramètre certainement plus héritable que la persistance.

Le coefficient de persistance hebdomadaire $\mathrm{CP}_{2 \div 3}$ est en corrélation légèrement négative avec les performances initiales $\mathrm{P}_{1}(r=-0,30)$, mais indépendant de la production totale. On obtient d'ailleurs le même résultat en remplaçant ce coefficient par le simple rapport entre les productions des $2^{\mathrm{e}}$ et $3^{\mathrm{e}}$ périodes $\left(\mathrm{P}_{2: 3}\right)$. Il est donc possible de calculer un taux de décroissance (ici, régression hebdomadaire de $7 \mathrm{p}$. IOo) indépendant du niveau de production total et de construire des courbes théoriques de lactation. On sait que chez les bovins, les travaux de Brody et al, Gaines et al, LEROY (r93I, I936), ont abouti à la détermination de courbes du même genre, traduisant une diminution mensuelle constante de Io p. Ioo de la production de lait à 4 p. roo de matière grasse.

\section{II. - Production laitière pendant l'allaitement}

Pour les sept premières semaines, nous disposons de 84 lactations : 43 de brebis allaitant I agnea11, 4I allaitant det1x agneaux.

Les contrôles hebdomadaires ne permettent pas d'estimer la production maximum journalière avec beaucoup de précision. Ils donnent cependant des résultats plus exacts que ceux déduits des courbes moyennes de lactation : le maximum observé étant inférieur de 2 à $300 \mathrm{~g}$ au maximum calculé, comme on peut s'en rendre compte d'après l'examen du tableau 3 et du graphique 2 .

Production maximum (tableau $3 b$ ). - Pour les brebis avec I et 2 agneaux, le maximum est respectivement de I,7I et $2,30 \mathrm{~kg}$, soit une différence de $34 \mathrm{p}$. Ioo.

De la I è la $2^{\mathrm{e}}$ lactation, on observe un accroissement de ro à 20 p. Ioo pour les deux catégories de brebis, mais aucune différence n'existe entre les $2^{\text {e }}$ et les lactations adultes.

Stade auquel intervient la production maximum. - Pour les brebis avec I ou avec 2 agneaux, le maximum se situe respectivement à Ig et 16 jours.

Avec le numéro de lactation, on n'observe aucune variation sensible. Par ailleurs, il n'existe pas de corrélation entre le niveau maximum et le stade auquel il intervient.

Courbes moyennes d'évolution de la production laitière. - Il existe en fait des différences dans l'évolution de la production avec le stade de lactation. En regroupant les données des $2^{\mathrm{e}}$ lactations et des brebis "adultes " ( $3^{\mathrm{e}}$ lactations, en majorité), on obtient les courbes moyennes du graphique 2 .

Pour les brebis en Ire lactation, élevant I et 2 agneaux, leur production présente une évolution parallèle (maximum à $\mathrm{I} 7$-I 8 jours) mais atteint un niveau différent.

Les bessonnières en $2^{\mathrm{e}}$ et $3^{\mathrm{e}}$ lactation ont un maximum élevé et précoce ( $4_{4}^{\mathrm{e}}$ jour), alors que pour les brebis du même âge, n'allaitant qu'un seul agneau, la courbe moyenne de lactation plafonne du $\mathrm{I}^{\mathrm{e}}$ au $35^{\mathrm{e}}$ (20 jours en moyenne).

Ces évolutions de la quantité de lait tété, qui confirment les observations de

Annales de Zootechnie. - I962. 


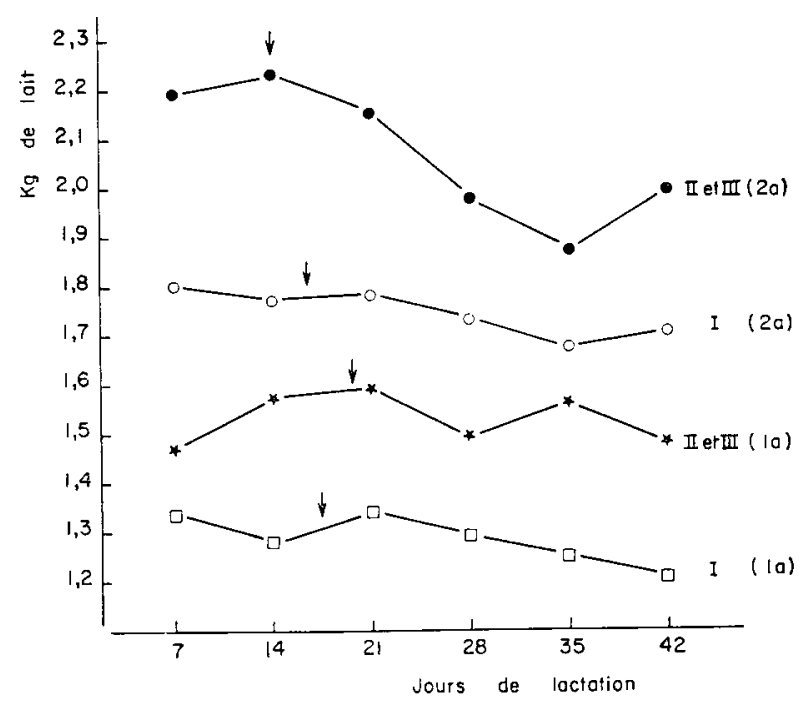

GRAPHIQUe 2.-- Evolution de la quantité de lait consomnépar les agneaux au cours de la période d'allaitement.

I. Ire lactation Ia: brebis avec $\mathrm{I}$ agneau

II. $2^{e} \quad-\quad 2 \mathrm{a}$ : brebis avec 2 agneaux.

$\downarrow$ Situation du maximum moyen journalier calculé d'après les contrôles hebdomadaires.

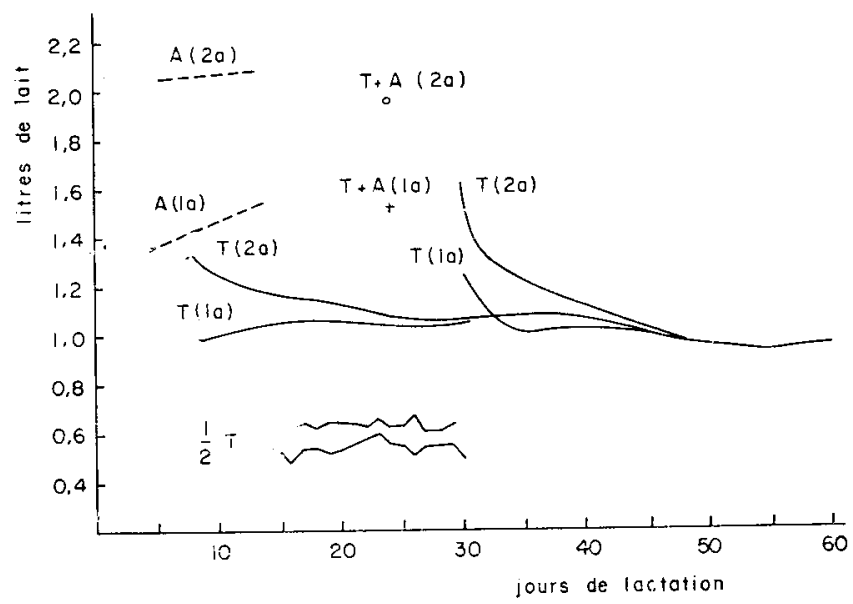

GraPhIQUE 3. - Production laitière des brebis sommises à l'allaitement, à la traite partielle el à la traite tolale, comparée à celle des brebis soumises à la traite complète 7 jours après la mise bas (Brouessy, 1959).

Légende : Ia : brebis avec I agneau;

2a: brebis avec 2 agneaux

A : Allaitement complet;

I/2T : Traite partielle au cours du sevrage progressif après $\mathbf{I} 2$ heures de séparation des agneaux.

$\mathrm{T}+\mathrm{A}: 12$ heures d'allaitement + traite partielle;

$\mathrm{T}$ Traite complète. 
BARNICOA'T et al. (I949), peuvent s'expliquer à la fois par la possibilité des agneaux jumeaux de téter tout le lait de leur mère, et par la meilleure aptitude laitière de cette dernière en $2^{\mathrm{e}}$ et $3^{\mathrm{e}}$ lactation.

Il importe également de souligner que si les bessonnières produisent en moyenne, au cours des 6 premières semaines, 37 p. Ioo de lait de plus que les brebis allaitant I seul agneau (tableau I) cette supériorité est constante et égale à $34 \mathrm{p}$. Ioo du Io ${ }^{\mathrm{e}}$ au $40^{\circ}$ jour de lactation pour les antenaises, alors qu'elle décroît de 45 à $27 \mathrm{p}$. Ioo au cours de la même période, pour les brebis en $2^{\mathrm{e}}$ et $3^{\mathrm{e}}$ lactation.

Pour les 6 premières semaines, 1'accroissement de production laitière de la $I^{\text {re }}$ à la $2^{\mathrm{e}}$ lactation est respectivement de 22 et $\mathrm{I} 8 \mathrm{p}$. Ioo pour les brebis allaitant $\mathrm{I}$ et 2 agneaux. Ces résultats sont comparables à ceux de BARxicoAt et al (I949, I956), STARKe (I953) Georgiou (I950). La plus grande part de cet accroissement doit être attribuée à la brebis et non aux agneaux ; l'augmentation du poids à la naissance du $\mathrm{I}^{\mathrm{er}}$ au $2^{\mathrm{e}}$ agnelage étant relativement faible $(3,7$ à $4, \mathrm{I} \mathrm{kg}$ pour les agneaux Préalpes simples - 6,2 à $6,4 \mathrm{~kg}$ pour les couples de jumeaux).

\section{III. - Période de traite succédant à l'allaitement. Influence des différents facteurs zootechniques sur la quantité de lait commercialisée.}

$L_{1}$ ? passage à la traite intervient da:s l'ensemble à un stade voisin du maximum de la production laitière. Les performanzes obtenues au cours des premières traites ne peuvent cependant être rattachées à la phase croissante de la lactation, car elles traduisent essentiellement l'adaptation de la brebis au régime de sevrage ou de traite.

\section{Mode de sevrage.}

a) Phase initiale de traite.

serrage brutal.

Les performances laitières obtenues au cours de la phase initiale d'allaitement sont supérieures à celles enregistrées à la traite depuis l'agnelage, mais cette amélioration disparaît après le sevrage. En effet, au bout de quelques jours, la production des brebis ayant allaité $\mathrm{I}$ ou 2 agneaux s'effond re au niveau de la lactation de référence.

Entre la dernière semaine d'allaitement et la I $^{\text {re }}$ semaine de traite complète, la chute est en moyenne de $365 \mathrm{~g}$ pour les brebis avec simples et de $600 \mathrm{~g}$ pour les bessonnières, soit respectivement 27 et $34 \mathrm{p}$. Ioo du lait tété (1) (tableau 6).

Au cours des 2 semaines consécutives au sevrage, l'évolution de la production laitière journalière se traduit par une diminution suivie d'une remontée avant la décroissance normale : la production de la $2^{\mathrm{e}}$ semaine de traite est donc approximativement équivalente à celle de la $\mathrm{I}^{\mathrm{re}}$ semaine. L'existence d'une phase croissante au cours de la $2^{\mathrm{e}}$ semaine résulte probablement de la suppression du stress provoqué par le sevrage et de l'adaptation progressive des brebis au régime de traite.

(I) Comme le sevrage se situe au début de la phase de décroissance de la lactation, on doit corriger l'importance de cette chute en tenant compte d'une régression hebdomadaire du niveau de production d'environ 7 p. 100 . La différence corrigée entre lait tété et lait trait (au même stade) n'est plus alors que de 22 et 30 p. 100 respectivement pour les brebis avec 1 et 2 atgneaux. 
G. RICORDEAU, R. DENAMUR

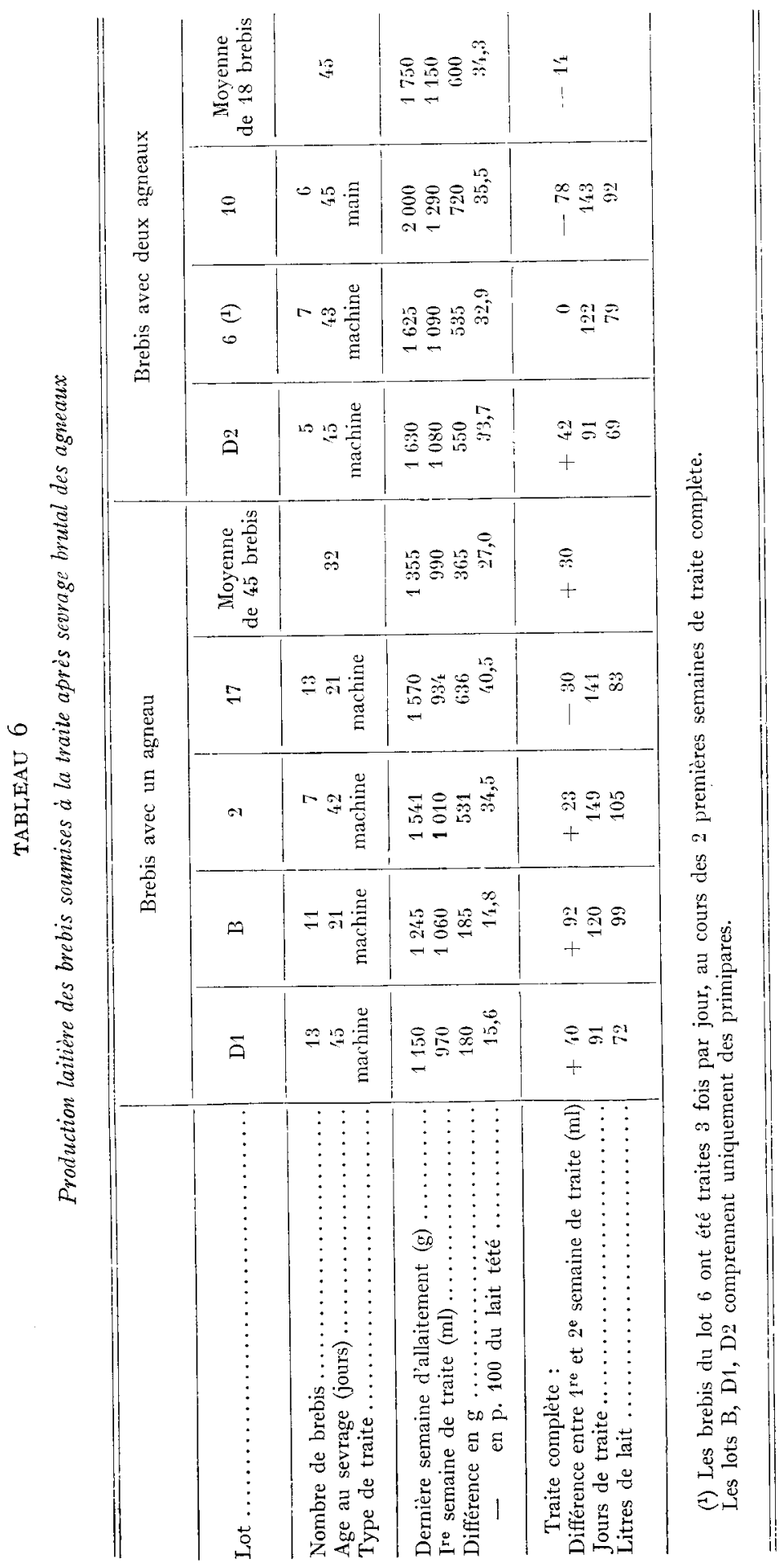


PRODUC'TION IAITIËE DES BREBIS

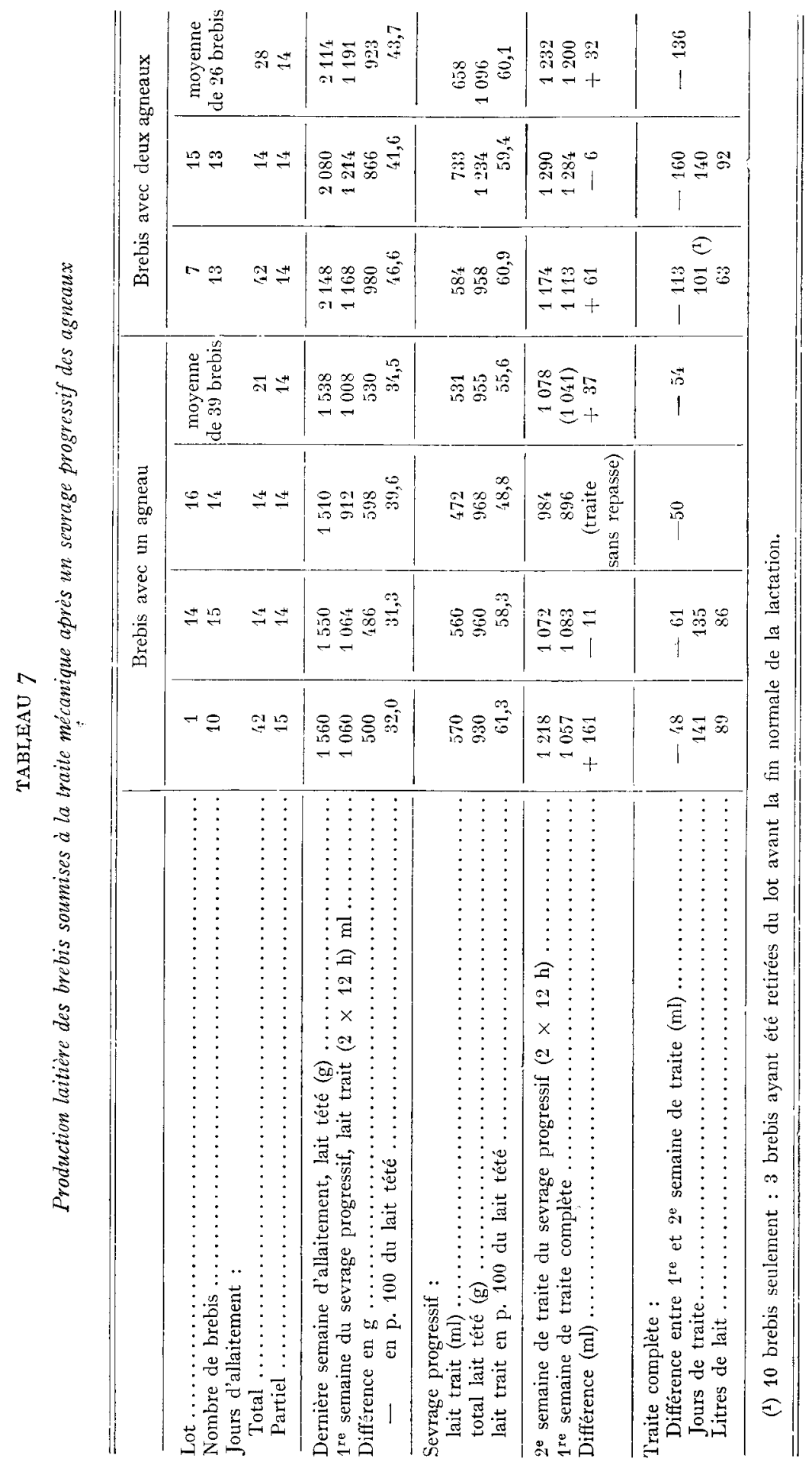


sevrage progressif.

En séparant progressivement les agneaux de leur mère, pour essayer de réduire l'importance de la rétention lactée, la production laitière des brebis s'établit 'à un niveau plus élevé, mais décroît de la $\mathrm{I}^{\mathrm{re}}$ à la $2^{\mathbf{e}}$ semaine de traite complète (différence de 50 et $140 \mathrm{ml}$ pour les brebis ayant allaité $\mathrm{I}$ et 2 agneaux respectivement - tableau 7 , graphiques 3 et 4 ) pour atteindre le même niveau que les brebis soumises au sevrage brutal.

b) Quantités totales de lait commercialisées. - Les comparaisons effectuées sur la même période de lactation dans le tableau 8 ne font apparaître aucune différence sauf en r959 où le sevrage progressif a donné des résultats supérieurs au sevrage brutal.

TABLEAU 8

Influcnce du mode de sevrage sur la production de lait commercialisable

\begin{tabular}{|c|c|c|c|c|c|c|c|}
\hline \multirow{4}{*}{$\begin{array}{l}\text { Node de sevrage } \ldots \ldots \ldots \ldots \ldots \\
N^{n} \text { lu lot } \ldots \ldots \ldots \ldots \ldots \ldots\end{array}$} & \multirow{2}{*}{\multicolumn{2}{|c|}{$\begin{array}{c}\text { Brouessy } 1958 \\
1 \text { agneaul }\end{array}$}} & \multirow{2}{*}{\multicolumn{2}{|c|}{$\begin{array}{l}\text { Jouy } 1958 \\
\text { atgneaux }\end{array}$}} & \multicolumn{3}{|c|}{ Bronlessy 1959} \\
\hline & & & & & \multicolumn{2}{|c|}{1 agrneau } & \multirow{3}{*}{$\mid \begin{array}{c}2 \text { agneaux } \\
\text { progressif } \\
15\end{array}$} \\
\hline & progressif & brutal & progrensif & brutal & progressif & brutal & \\
\hline & 1 & 2 & 7 & 6 & 14 & 17 & \\
\hline Nolnbre de brebis ........... & 10 & 7 & $1: 3$ & i; & 15 & $1: 3$ & 13 \\
\hline \multicolumn{8}{|l|}{ Durée d'allaitement : } \\
\hline complet $\ldots \ldots \ldots \ldots \ldots \ldots$ & 42 & 42 & 42 & 价 & $1^{\prime} t$ & 21 & $11^{\prime}$ \\
\hline particl $\ldots \ldots \ldots \ldots \ldots \ldots$ & 15 & 0 & 1it & 1) & 15 & 0 & l't \\
\hline \multicolumn{8}{|l|}{ Lait trait au cours } \\
\hline du sevrage jrogressif (litres) ${ }^{(1)}$ & 17 & - & 16 & $\cdots$ & 8 & $\ldots$ & 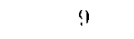 \\
\hline \multicolumn{8}{|l|}{ Traite complite: } \\
\hline litres de lait ............. & 89 & $10 \%$ & 1i:3 & 79 & $\therefore 6$ & s:: & $9 \cdot 2$ \\
\hline durće (jours) . . . . . . . . & $1 \div 1$ & 149 & 101 & $1 \div 2$ & $1: 5$ & 111 & $1: 3$ \\
\hline \multicolumn{8}{|l|}{ Période de rérence : } \\
\hline litres de lait $\ldots \ldots \ldots \ldots \ldots$ & 106 & $10^{\prime}$ & 79 & 79 & $9^{\prime}$ & s:3 & 101 \\
\hline churée (jouss) ............ & 156 & 149 & 115 & $1 \geq 2$ & $14: 3$ & 111 & $19=$ \\
\hline Différence (litres) & + & & 1 & & $\therefore$ & & \\
\hline
\end{tabular}

(1) Estimation obtenue en doublant le résultat de la traite particlle.

A l'exception de la phase initiale de traite, le sevrage progressif ne permet donc pas d'améliorer la production de lait commercialisée.

$2^{\circ}$ Nombre d'agneaux nés ou allaités.

Nous avons comparé de nombreux lots de brebis ayant allaité I et 2 agneaux. Il nous est cependant difficile de tenir compte de tous les résultats. D'une part, les lots n'ont pas toujours été placés dans la même bergerie, d'autre part, la fréquence des mammites a été pius à élevée pour la catégorie des bessonnières (30 p. Ioo environ en I957 et I958). Les brebis des lots restant à la traite sont donc non seulement en faible effectif, mais représentent un échantillon " biaisé ". Nous avons montré par exemple, dans l'étude spéciale relative aux mammites que les brebis infectées produisaient au moment de l'infection, I20 à I30 p. Ioo de la production moyenne du lot restant à la traite. 
Pour nos comparaisons, nous retiendrons donc uniquement les résultats de 2 couples de lots entretenus à Brouessy en I959 (tableau 9). Dans les 2 cas, pour l'ensemble de la période de traite, la différence est de 3 à 6 litres en faveur des bessonnières. En fait, cette différence est celle enregistrée uniquement lors des 2 premières semaines consécutives au sevrage définitif. D'après le graphique 3 par exemple, il ressort nettement que la production laitière des bessonnières s'établit à un niveau élevé, puis régresse rapidement au niveau de celles ayant élevé des simples.

TABI,EAU 9

Infuence du nombre d'agneaux nés ou allaités sur les performances laitières à la traite

\begin{tabular}{|c|c|c|c|c|}
\hline \multirow[b]{2}{*}{$\begin{array}{l}\text { Nombre de brebis } \ldots \ldots \ldots \ldots \ldots \\
\text { Annelée } \ldots \ldots \ldots \ldots \ldots \ldots \ldots \ldots\end{array}$} & \multicolumn{2}{|c|}{$\begin{array}{c}\text { Traite apres } 6 \text { jours } \\
\text { d'allaitement } \\
\text { (lots } 11 \text { et } 12 \text { ) }\end{array}$} & \multicolumn{2}{|c|}{$\begin{array}{c}\text { Traite après } 15 \text { jours } \\
\text { d'allaitement complet } \\
\text { et } 15 \text { jours d'allaitement partiel } \\
\text { (lots } 1 \text { 't et } 15)\end{array}$} \\
\hline & $\begin{array}{r}10 \\
1\end{array}$ & $\begin{array}{r}21 \\
2\end{array}$ & $\begin{array}{r}15 \\
1\end{array}$ & $\begin{array}{r}13 \\
2\end{array}$ \\
\hline $\begin{array}{l}\text { Jours de lactation } \ldots \ldots \ldots \ldots \ldots \ldots \\
\text { Litres de lait } \ldots \ldots \ldots \ldots \ldots \ldots \ldots \\
\text { Différence } \ldots \ldots \ldots \ldots \ldots \ldots \ldots\end{array}$ & $\begin{array}{l}168 \\
109,6\end{array}$ & $\begin{array}{r}159 \\
119,6 \\
+\quad 3,0 \\
\end{array}$ & $\begin{array}{l}165 \\
85,6\end{array}$ & $\begin{array}{r}170 \\
91,6 \\
+\quad 6,0 \\
\end{array}$ \\
\hline
\end{tabular}

$3^{\circ}$ Durée de la période d'allaitement.

Les comparaisons effectuées dans le tableau Io, sur les mêmes périodes de lactation, indiquent que la durée d'allaitement ne modifie pas les performances de la brebis, ni en production commercialisée, ni en persistance. En 1957, comme en I959, les lots de bessonnières ou de brebis avec I agneau, sevrés à $2 \mathrm{I}, 28,42$ ou 49 jours, donnent (pour une même période de lactation) des productions tout à fait comparables aux autres lot soumis à la traite quelques jours après la mise bas. I1 est d'ailleurs intéressant de vérifier qu'en I958 les lots 4 et 5 , contrôlés pendant l'allaitement après séparation des agneaux, ont donné des performances équivalentes à celles des lots 9 ( $\mathrm{r}$ re et $2^{\mathrm{e}}$ lactation), dont les brebis avaient été soumises à la traite quelques jours après la misè bas.

En résumé, on peut dire qu'il existe une courbe de base (lactation de référence) sur laquelle on retombe toujours quand on passe à la traite et cela quel que soit le mode de sevrage utilisé, le niveau de production laitière à la tétée (brebis ayant mis bas $\mathrm{I}$ et 2 agneaux) et la durée de la période d'allaitement.

\section{IV. - Efficacité comparée de la traite et de la tétée}

Les résultats précédents ont mis en évidence l'existence d'une différence de production entre les brebis allaitantes et les brebis traites. Pour préciser 1'importance et les causes de cette différence, nous avons effectué sur 3 groupes de brebis 2 séries de contrôles : allaitement suivi de traite, alternance de tétées et de traites.

$I^{\text {er }}$ groupe : I 8 brebis avec I agneau et 36 bessonnières, séparées de leurs agneaux (en moyenne à 23 jours) pendant 24 heures et traites manuellement 3 fois par jour (6, I 2 et I 8 heures). 


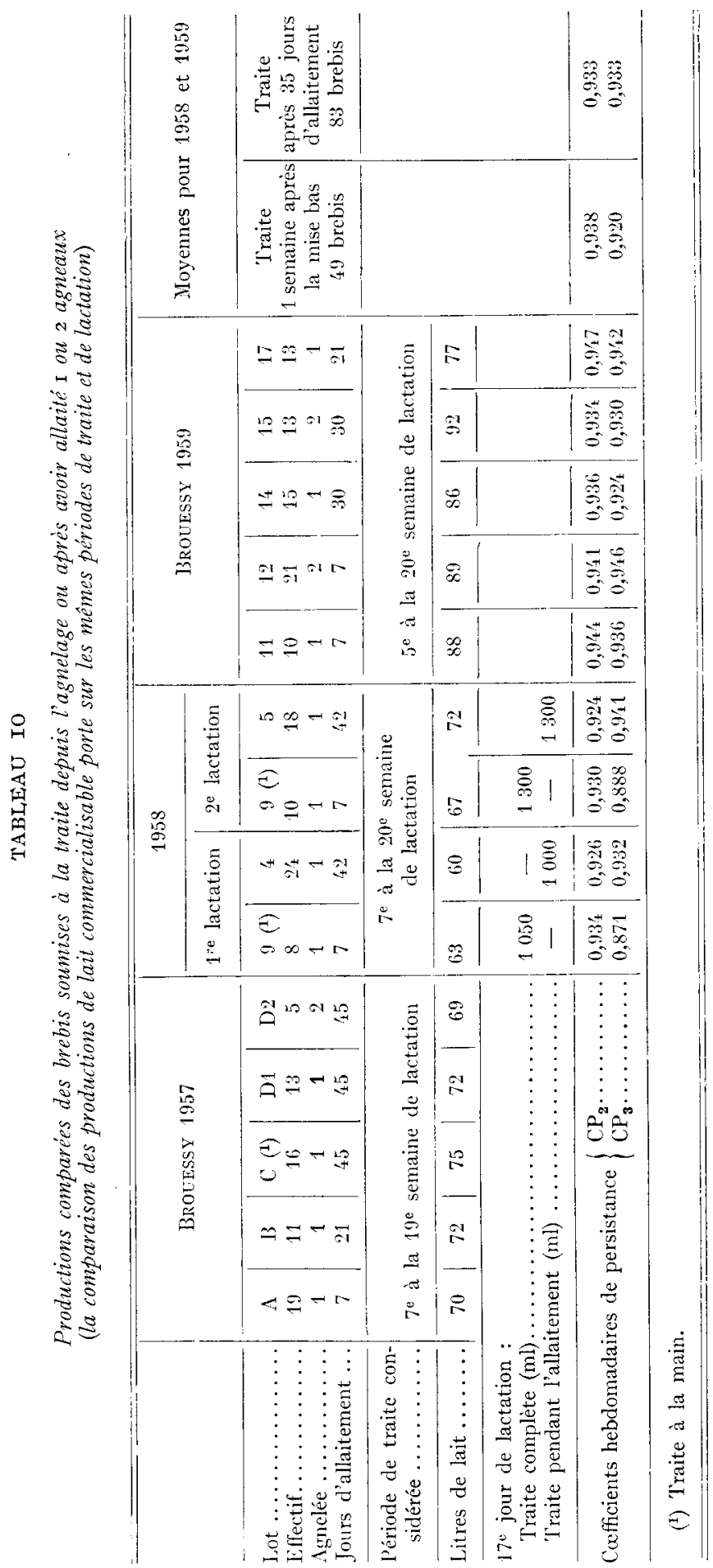




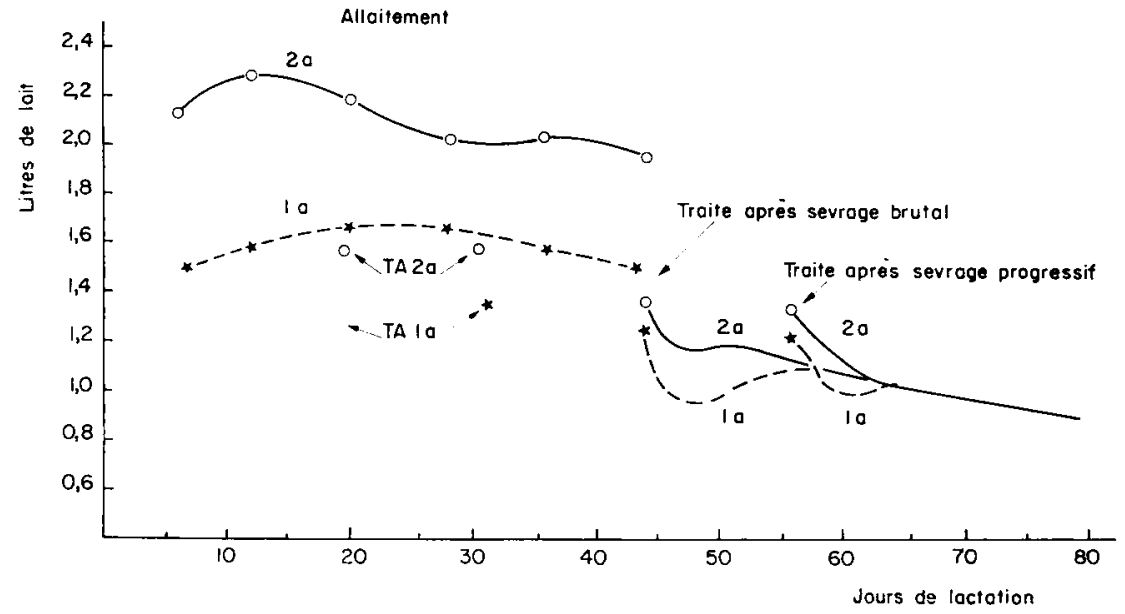

GRAPHIQLE 4. - I'roduction laitière des brebis sommises a la traite après an sevrage brital ou progressit des agneaux (Jouy-et-Brouessy, 1958).

Ia : brebis avec I agneau; $2 \mathrm{a}$ : brebis avec 2 agneaux; A : allaitement complet ; TA : traite pendant l'allaitement après séparation des agneaux pendant 24 heures; $\mathrm{T}$ : traite complète;

Après 40 jours d'allaitement complet, les brebis avec 1 et 2 agneaux ont été réparties en 2 lots :

I er lot : 2 traites par jour après séparation définitive des agnéctux;

$2^{\mathrm{e}}$ lot : 2 traites par jour après une période intermédiaire de 2 semaines correspondant au sevrage progressif des agneaux (allaitement et traite en alternanes).

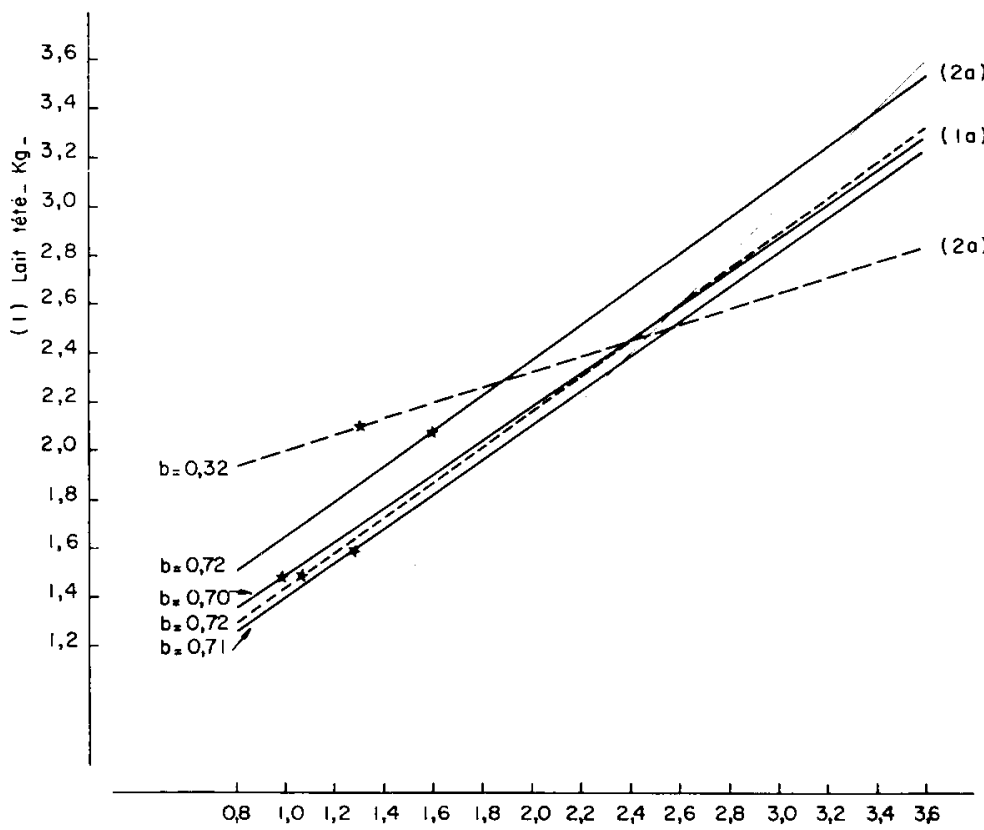

(2) Loit trait - $\mathrm{Kg}-$

GRAPHIQUe 5. -- Régressions des quanités de lait obtenues à la télée sur les quantités de lait obienues à la traite.

Les lignes de régression correspondent aux 5 lots du tableau II. En trait plein : traite après allaite* ment (sevrage brutal); lin trait pointillé : traite et allaitement (sevrage progressif); ra: brebis avec $r$ agneau; $2 a$ : brebis avec 2 agneaux. 


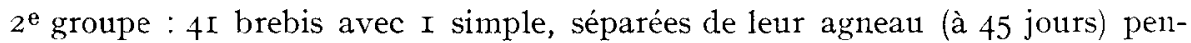
dant 48 heures, et traites 2 fois par jour à la machine.

$3^{\mathrm{e}}$ groupe : 47 brebis allaitant $\mathrm{I}$ agneau et 32 bessonnières soumises durant $\mathrm{I} 4$ jours au sevrage progressif : alternance de I 2 heures d'allaitement ( $8 \mathrm{~h}$ à $6 \mathrm{~h}$ ) et de I2 heures de séparation ( $6 \mathrm{~h}-\mathrm{I} 8 \mathrm{~h})$ terminées par la traite à $\mathrm{I} 8 \mathrm{~h}$.

Le lait tété est estimé :

- pour le I $^{\text {er }}$ groupe, par la moyenne des 2 contrôles d'allaitement effectués avant et après la journée de traite.

- pout le $2^{\mathrm{e}}$ groupe, par la moyenne des contrôles d'allaitement effectués au cours des 2 jours précédant les journées de traite.

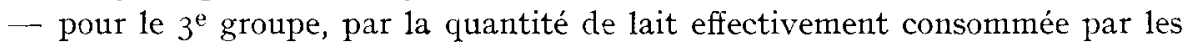
agneaux pendant une période nocturne de $I 2$ heures, la portion de lait récupérée par la tétée des agneaux immédiatement après la traite manuelle étant exclue.

A chaque contrôle d'allaitement, la quantité de lait tété a été estimée par pesée des agneaux toutes les 2 heures sur une période de $r 2$ heures.

Le tableau I I résume les résultats obtenus à partir de ces 3 groupes.

I) Dans les 3 groupes, les performances d'allaitement sont comparables, mais les performances de traites sont supérieures pour les brebis du I $^{\text {er }}$ groupe, séparées

TABI,EAU II

Comparaison des quantités de lait obtenues à la tétée et à la traite

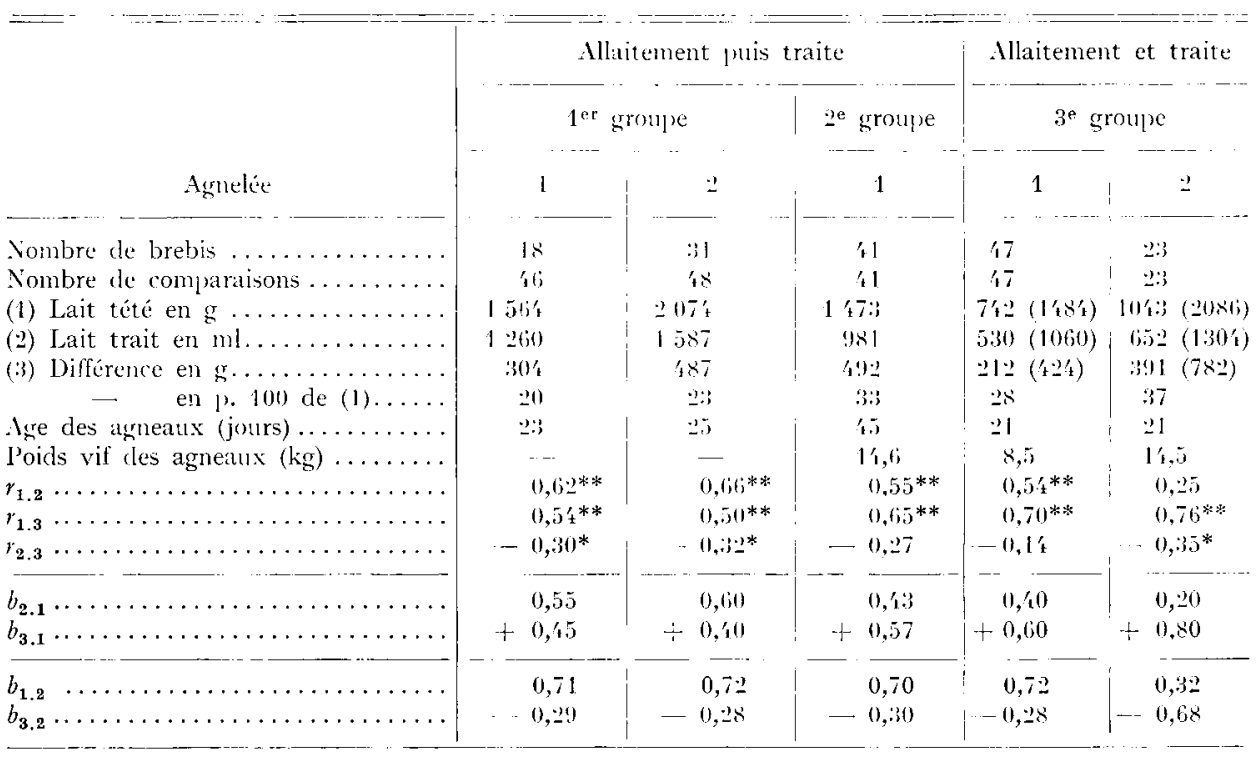

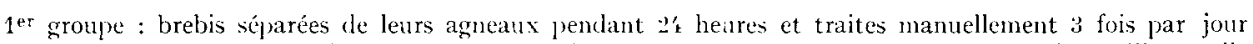
$(3,6$ et $18 \mathrm{~h})$. Ces brebis ont été traites sur 2 ou 3 périodes de 24 heures à 10 jours d'intervalle environ, à jartir de la $1^{\text {re }}$ semaine de lactation.

$2^{\text {e }}$ groule : brebis sćparées de leur agneau pendant is heures et traites 2 fois par jour à la machine.

$3^{\text {e }}$ groupe : brebis soumises au sevrage progressif : I? heures clallaitement et traite après 1: heures re séparation.

** Significatif an senil $\mathrm{P}=0,01$.

* Sirnificatif an seuil $\mathrm{P}=0,05$. 
pendant 24 heures de leurs agneaux et traites manuellement 3 fois par jour. Il est vraisemblable que cette traite supplémentaire est responsable de la différence observée.

2) Il existe une corrélation positive de l'odre de o,60 entre la quantité de lait trait et la quantité de lait tété ; la relation est cependant plus faible pour les bessonnières du $3^{\mathrm{e}}$ groupe.

3) La traite sous-estime 1'aptitude laitière:

- de 20 et 23 p. Ioo dans le cas de 3 traites manuelles en 24 heures;

- de $33 \mathrm{p}$. Ioo dans le cas de 2 traites à la machine ;

- de 28 et 37 p. Ioo dans le cas de la traite quotidienne à la machine en cours de sevrage progressif.

4) La différence entre quantités de lait tété et de lait trait (paramètre susceptible d'être retenu comme critère de facilité de traite) est en corrélation positive étroite avec la I $^{\mathrm{re}}$ de ces variables et en corrélation légèrement négative avec la seconde.

5) Cette différence est en relation positive avec le poids vif des agneaux, pour les animaux du $\mathrm{I}^{\mathrm{er}}$ et $\mathrm{du} 3^{\mathrm{e}}$ groupe contrôlés entre le $\mathrm{I} \mathrm{o}^{\mathrm{e}}$ et le $3 \mathrm{O}^{\mathrm{e}}$ jour de lactation (poids moyen : $8 \mathrm{~kg}$ ) ; elle est pratiquement indépendante de leurs poids lorsque cruxci ont atteint un développement suffisant (poids moyen : I5 $\mathrm{kg}$ ) et sont capables de téter tout le lait produit par leur mère (agneaux simples du $2^{\text {e }}$ groupe et jumeaux des $I^{\text {er }}$ et $3^{\mathrm{e}}$ groupe).

6) Compte tenu de la relation positive entre les quantités tétées et les quantités traites, il résulte statistiquement que tout accroissement de l'aptitude laitière à la tétée ( + roo g par exemple pour les brebis du $2^{\text {e }}$ groupe dont l'agneau a été sevré brutalement à 45 jours) s'accompagne d'un accroissement inférieur de l'aptitude laitière à la traite $(+43 \mathrm{~g})$, mais aussi d'une augmentation correspondante de la différence $(+57 \mathrm{~g})$.

Au contraire, tout accroissement du niveau de production à la traite $(+$ Ioo $\mathrm{g}$ toujours sur le même exemple) se traduit par une amélioration importante de lait tété $(+70 \mathrm{~g})$ et une diminution correspondante de la différence (-30 g).

Di1 point de vue des corrélations phénotypiques, si l'on veut améliorer l'aptitude laitière à la traite et augmenter encore l'aptitude laitière à la tétée tout en réduisant l'écartentre ces deux performances, il convient donc de sélectionner essentiellement surles performances de traite. D'après l'intersection des différentes lignes de régression avec la bissectrice (graphique 5), on peut même prévoir que les performances de traite seront supérieures en moyenne à celles enregistrées à la tétée, lorsqu'elles dépasseront une valeur de l'ordre de 2,3 litres pour les brebis avec I agneau et 3,4 litres pour les bessonnières. Ces limites correspondent d'ailleurs à la capacité d'absorption maximum de la moyenne des agneaux.

\section{V. - Problèmes posés par le sevrage progressif}

Pendant les 2 semaines de sevrage progressif, le fait de laisser les agneaux avec leur mère pendant la nuit et de les séparer ensuite jusqu'à la fin de la traite quotidienne, permet-il d'obtenir une production de lait comparable en quantité et qualité à celle obtenue lors de la traite complète? 
I $^{\circ}$ Aspect quantitatif (tableau I2)

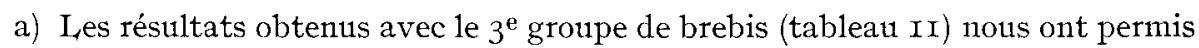
d'établir la correspondance entre les quantités de lait obtenues à la traite (2) et à la tétée en $\mathrm{I} 2$ heures ( $\mathrm{I}$ ). Il est intéressant d'ajouter que si la différence (3) entre ces 2 quantités est en corrélation légèrement négative avec le niveau de production enregistré à la traite au cours du sevrage progressif, elle est pratiquement indépendante:

- du niveau de production manifesté lors de la phase initiale de traite complète (IO) ;

- de la quantité de lait obtenue à la repasse au cours des périodes de sevrage et de traite complète (7 et II).

b) Compte tenu des intervalles de sécrétion, le volume de lait obtenu à la traite pendant le sevrage progressif (2) est une bonne indication de l'aptitude laitière manifestée lors des premiers jours de traite complète. Pour les brebis allaitant des simples, les corrélations entre ces paramètres $\left(r_{28}\right.$ et $r_{29}$, tableau I2$\left._{2}\right)$ sont de l'ordre de 0,75 (c'est-à-dire comparables à celles qui existent entre les performances $\mathrm{du} 4^{\mathrm{e}}$ et $\mathrm{du} \mathrm{Io}$ jour de traite complète) alors qu'avec les bessonnières les corrélations obtenues sont plus faibles $(0,40)$ par suite peut-être d'une plus grande difficulté d'adaptation des brebis à la traite mécanique.

c) I a quantité de lait tété au cours de la période nocturne ne représente pas la totalité du lait consommé par les agneaux. En effet, pour réaliser une vidange plus complète de la mamelle et estimer aussi précisément que possible la sécrétion de lait pendant les $\mathrm{I} 2$ heures où les mères allaitent leurs agneaux, ces derniers sont mis en présence de leur mère dès la fin de la traite. I a vidange ainsi obtentue (4) représente en moyenne $3 \mathrm{I}$ p. Ioo du volume total de lait obtenu à la traite pour les 2 catégories de brebis. Son importance est en corrélation positive avec le poids vif des agneaux mais indépendante du niveau de production laitière enregistré à la traite machine ou à la repasse. Si Eyal, Volcani et Sharav (I958) observent également que la tétée des agneaux après l'égouttage manuel permet d'obtenir en moyenne $23 \mathrm{p}$. Ioo de la production totale journalière (de II à $57 \mathrm{p}$. Ioo), ils constatent par contre une corrélation négative entre les quantités de lait obtenues à la traite et par l'agneau.

d) En tenant compte du lait récupéré par les agneaux immédiatement après la traite, l'écart entre la quantité totale de lait tété (5) et la quantité de lait trait (2) atteint 39 et $49 \mathrm{p}$. Ioo pour les 2 catégories de brebis.

$2^{\circ}$ Aspect qualitatif du sevrage progressif.

L'inefficacité de la traite quotidienne par rapport à la tétée se traduit également par l'obtention d'un lait appauvri en matière grasse, comme l'avait constaté Bosc (I958) sur des brebis de race Lacaune.

Nous avons pu confirmer et compléter cette observation (tableau I3). Lors de la $2^{\mathrm{e}}$ quinzaine de lactation, la richesse moyenne en matière grasse des laits de l'unique traite quotidienne s'établit à 4,5 contre 6,9 p. roo pour les laits des brebis soumises à la traite complète depuis l'agnelage. Dès la fin du sevrage, le taux butyreux des laits de mélange des lots sevrés progressivement remonte d'ailleurs à $7,0 \mathrm{~g}$. Fin ce qui concerne la teneur en matières azotées on enregistre peu de différences suivant le mode de traite. Aussi, le rapport matières azotées/matière grasse des laits de mélange du sevrage progressif est-il nettement plus élevé que le rapport normal (, 3 contre 
PRODUCTION IAITIËRE DES BREBIS

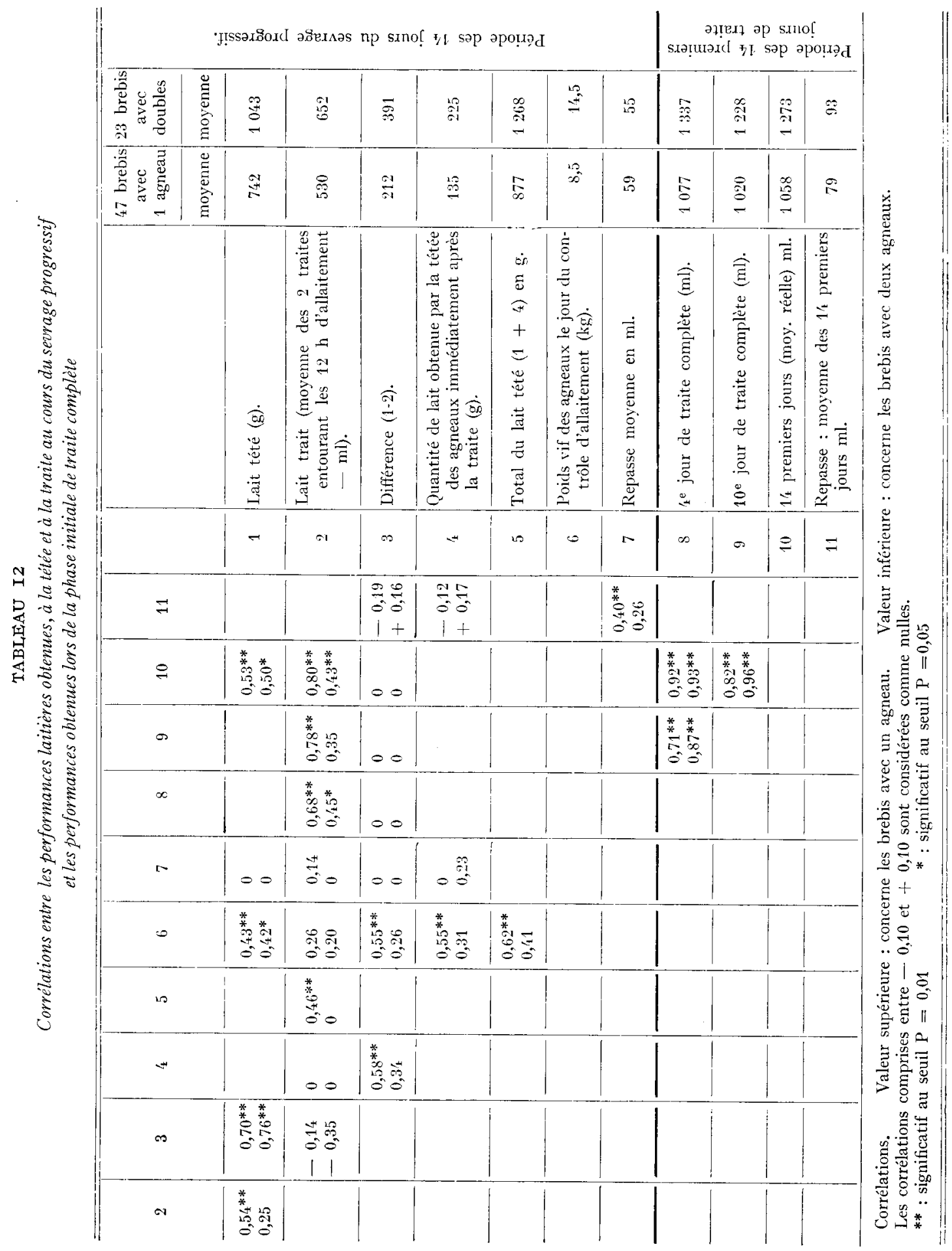


G. RICORDEAU, R. DENAMUR

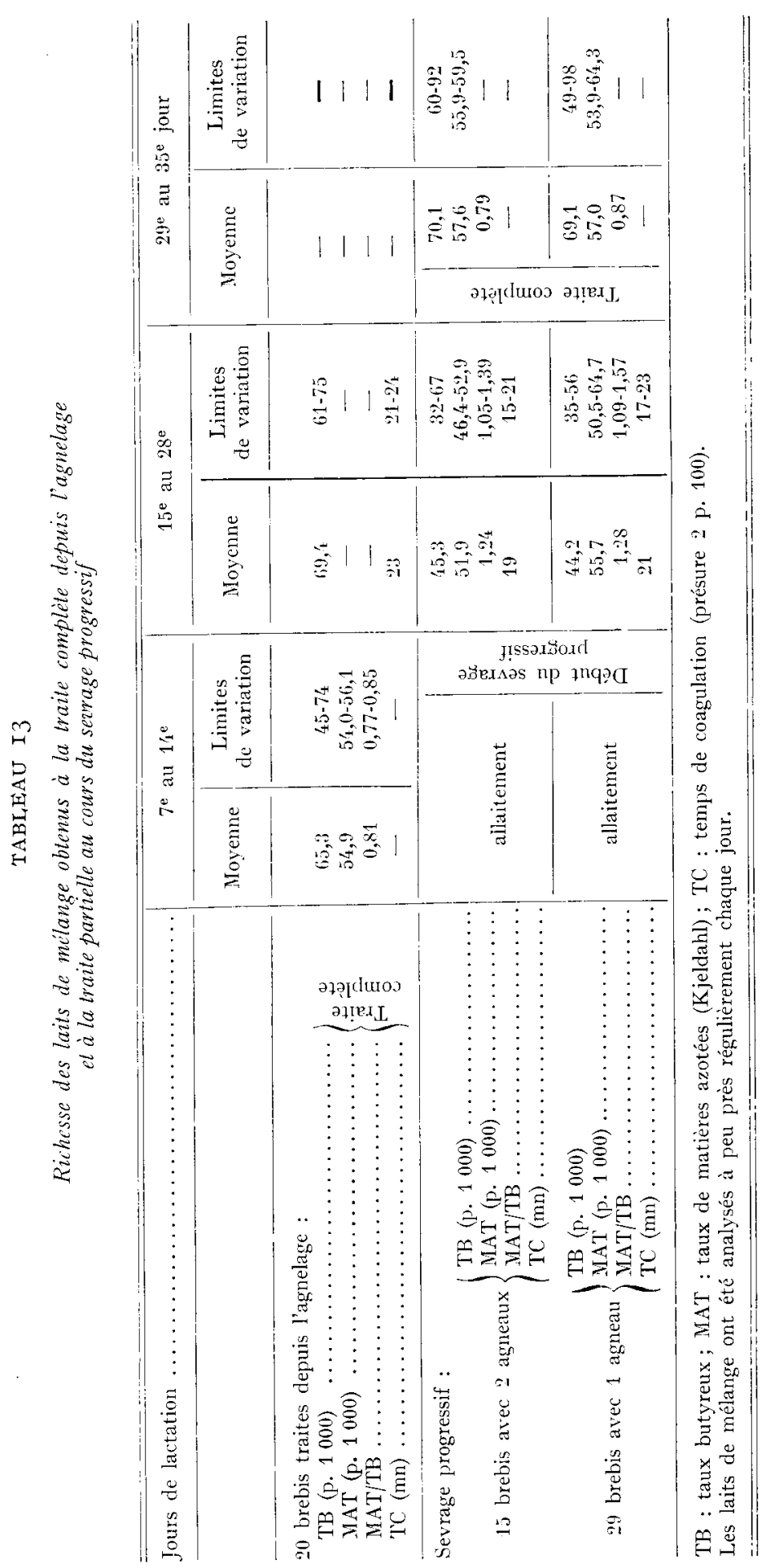


o,8). A défaut de tests précis sur la qualité des fromages pouvant être fabriqués avec ces laits appauvris en matière grasse, nous avons effectué quelques essais de coagulation. S'il n'apparaît pas de différences sensibles, les laits de sevrage progressif semblent coaguler légèrement plus vite, bien cu'aucune relation ne puisse être mise en évidence entre le rapport MAT/TB et le temps de coagulation.

\section{$3^{\circ}$ Infuence de la présence de l'agneau sur la descente du lait lors de la traite en cours de sevrage progressif.}

En laissant l'agneau en présence de sa mère, peut-on modifier le comportement de la brebis et obtenir une traite plus complète? Afin de répondre à cette question, nous avons contrôlé l'écoulement du lait de ro brebis dont 6 bénéficiaient de la présence $d=$ leur agneau (tableau I4, graphi(que 6).
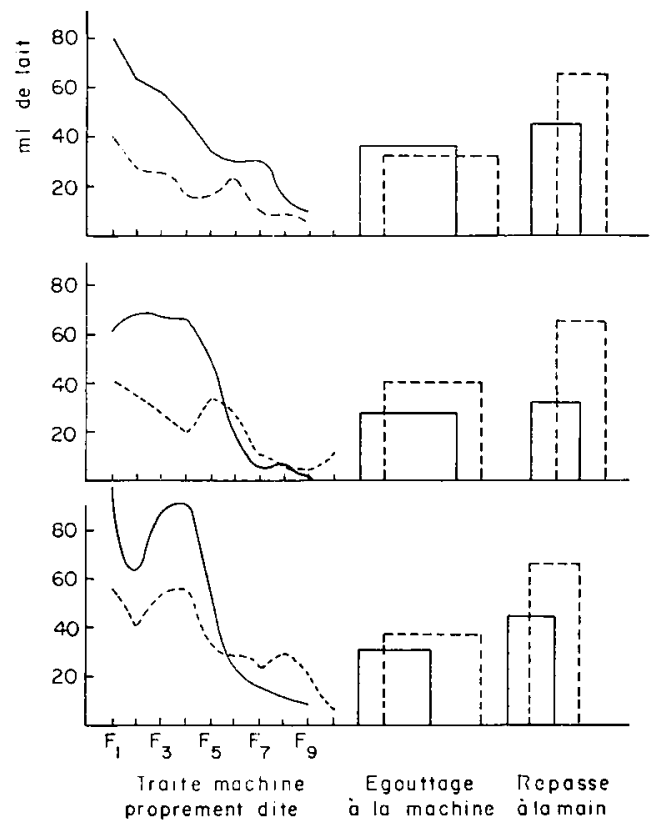

(inAPHove 6. - Ecoulement du lat au cours de la tratle du searage progressif.

....-.- brebis traite en l'absence de son agneau (4 brebis);

_-_ brebis traite en présence de son agneau ( 6 brebis) ;

La durée de la repasse manuelle a été estimée à zo serondes en moyenne;

lin haut $: 9^{\mathrm{e}}$ et $10^{\mathrm{e}}$ jour du sevrage progressif;

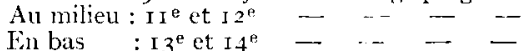

Pour les brebis traites en présence de leur agneau, il apparaît nettement que la descente de lait est beaucoup plus rapide et plus importante au cours de la première minute de traite machine. En comparant ces observations à celles que nous avons enregistrées par ailleurs lors de la période de traite succédant à l'allaitement (RICORDEAU, DENAMUR, MARTINET, I962) nous pouvons constater que la présence de l'agneau, non seulement ne retarde pas l'écoulement du lait citernal, mais assure en quelques jours un bien meilleur réflexe d'éjection $\left(^{1}\right)$.

(r) Ce résultat est confirmé par le comportenent de la brebis qui continue de ruminer lorsqu'on lui laisse son agneau. 


\section{TABLEAU I4}

Écoulement du lait au cours de la traite du sevrage progressif, en l'absence ou en présence de l'agneau

\begin{tabular}{|c|c|c|c|c|c|c|}
\hline \multirow[b]{3}{*}{ Jours du sevrage progressif $\ldots . \ldots \ldots \ldots \ldots \ldots \ldots$} & \multirow{2}{*}{\multicolumn{3}{|c|}{ 'Traite sans arrneau }} & \multicolumn{3}{|c|}{ Traite avec agneau } \\
\hline & & & & \multicolumn{3}{|c|}{6 brebis } \\
\hline & $9-110$ & $11-12$ & $13-1 t^{\prime}$ & $9-10$ & $11-12$ & $13-14$ \\
\hline \multicolumn{7}{|l|}{ Traite machine proprement dite: } \\
\hline lait $(\mathrm{ml}) \ldots \ldots \ldots \ldots \ldots \ldots \ldots$ & 202 & 230 & 342 & 372 & 337 & 460 \\
\hline 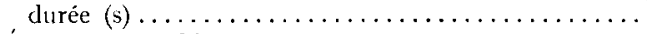 & $1: 8$ & 117 & 135 & 109 & 103 & 117 \\
\hline \multicolumn{7}{|l|}{ Égouttage machine : } \\
\hline lait $(\mathrm{ml}) \ldots \ldots \ldots$ & 181 & 161 & 148 & 140 & $10^{\prime}$ & 96 \\
\hline durée $(s) \ldots \ldots \ldots \ldots \ldots \ldots \ldots \ldots$ & 81 & 60 & 59 & 59 & $5 t$ & 45 \\
\hline Frgouttage manuel ou repasse $(m 1) \ldots \ldots \ldots \ldots \ldots$ & $s 0$ & 57 & 89 & 28 & 47 & 38 \\
\hline Total traite....... & $46: 3$ & 148 & 579 & 540 & 488 & 584 \\
\hline Lait tété par les aşneaux après la traite $(\mathrm{r})$. & $1: 99$ & $1 \geq 9$ & 132 & 88 & 65 & 89 \\
\hline Traite to tétée & 502 & 577 & 711 & $6 i 28$ & 5.3 & 673 \\
\hline
\end{tabular}

Chaque contrôle est la moyenne des résultats obtemus sur 2 jours consécutifs

Les agneaux étaient placés peu de temps avant la traite dans une stalle adjacente à celle des brebis.

Dans les 2 groupes, les agnéaux tétaient leur mère après la repasse manuelle en vue d'estimer l'efficacité de la traite.

Pour les brebis traites sans agneau, on enregistre corrélativement une quantité de lait plus importante, non seulement à l'égouttage machine et à la repasse, mais également à la vidange réalisée en fin de traite par les agneaux. Les productions totales sont finalement comparables.

Du point de vue de la traite, il importe de retenir :

- que la brebis est capable dans une certaine mesure de " contrôler " les mécanismes physiologiques d'évacuation du lait. La présence de l'agneau modifie son comportement dans des proportions cependant plus faibles que celles indiquées par Constantinescu et Gondos.

-. que les stimuli dus à la présence de l'agneau ne sont pas aussi efficaces sur l'éjection du lait que ceux fournis par la tétée elle-même.

\section{$4^{\circ}$ traite après injection de $\mathrm{x} U I$ d'ocytocine}

La tétée des agneaux immédiatement après la repasse manuelle, bien que représentant $3 \mathrm{I}$ p. Ioo du volume de la traite partielle du sevrage progressif, sous-estime encore le volume résiduel tel qu'on peut le déterminer en effectuant une traite supplémentaire après injection d'ocytocine. En effet, la quantité de lait obtenue après cette injection est supérieure de $240 \mathrm{~g}$ à celle obtenue par la tétée de l'agneau (tableau I5). Ces résultats correspondent d'ailleurs à ceux de Coombe et al. (rg6o) et aux observations que nous avons effectuées sur des brebis Texel (RICORDEAU-DENAMUR, I962). 
TABIEAU I 5

Efficaciti de la tétée par rapport à la traite apres injection d'ocytocine - brebis avec deux agneaux, traite pendant le sevrage progressif

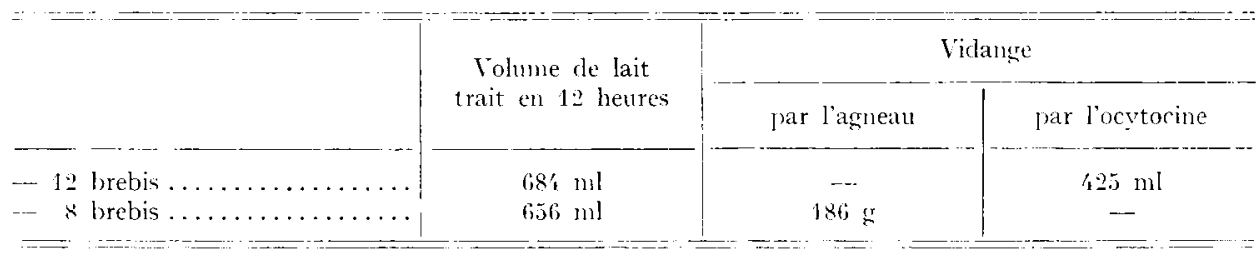

\section{DISCUSSION}

Io Sur le graphique 7 nous avons comparé les productions laitières des brebis soumises à la traite après une période d'allaitement plus ou moins courte, à celles qui allaitent leurs agneaux en permanence. Pour représenter ces dernières, nous avons retenu les données de Bonsma (I944), WAIIACE (I948), HUNTER (I956) et VERA Y VEGA (I956), relatives aux brebis Mérinos, Suffolk, Border Leicester et Manchegas. Les références des brebis soumises à la traite sont celles de Fincr, d'OGNJaxovic, de GEORgIOU, ainsi que les notres.

Il apparait nettement que la décroissance de la lactation des brebis allaitantes est plus rapide que celle des brebis traites à partir du $25^{\circ}$ jour d'allaitement. Cela est particulièrement net lorsque l'on compare, par exemple, la persistance des brebis Suffolk et Préalpes, dont les performances initiales sont équivalentes.

$2^{\circ}$ L'étude de la phase croissante de la lactation dans le cas d'allaitement on de traite quelques jours après la mise bas (lactation de référence) a permis d'établir que le maximum de production obtenu à la tétée est nettement supérieur à celui obtenu à la traite (4I, 54 et $39 \mathrm{p}$. Ioo pour les brebis avec simples en $\mathrm{I}^{\mathrm{re}}, 2^{\mathrm{e}}$ et $3^{\mathrm{e}}$ lactation, Ir et $6 \mathrm{r} \mathrm{p}$. Ioo pour les bessonnières en $2^{\mathrm{e}}$ et $3^{\mathrm{e}}$ lactation), mais se situe sensiblement au même stade (I 7 à 28 jours).

$3^{\circ}$ Le passage de $1^{\prime}$ allaitement à la traite a lieu habituellement vers 35 jours. Il intervient donc à un stade où la production est encore très élevée (3I p. Ioo des brebis avec I agneau et $\mathrm{I} 7 \mathrm{p}$. Ioo des bessonnières présentent un maximum entre 25 et 45 jours). Cette observation n'explique cependant pas pourquoi le sevrage brutal ou progressif se traduit par une rétention importante de la sécrétion qui ramène la production laitière obtenue à la traite au niveau de la lactation de référence (traite depuis la mise bas) et cela, quels que soient le nombre d'agneaux nés ou élevés et la durée d'allaitement.

Entre brebis allaitant I et 2 agneaux la différence de production laitière ne s'observe donc que pendant la période d'allaitement et à un degré moindre, lors des premiers jours de traite complète (notamment après un sevrage progressif). Ces résultats confirment les observations de MASOx et DASSAT (r953) et d'AI.EXANDER et Davies (I959).

$4^{0}$ La comparaison des performances laitières à la traite et à la tétée aul moment du sevrage brutal ou en cours de sevrage progressif a montré que si l'écart entre lait tété et lait trait était en corrélation positive avec la quantité de lait tété, il était par 


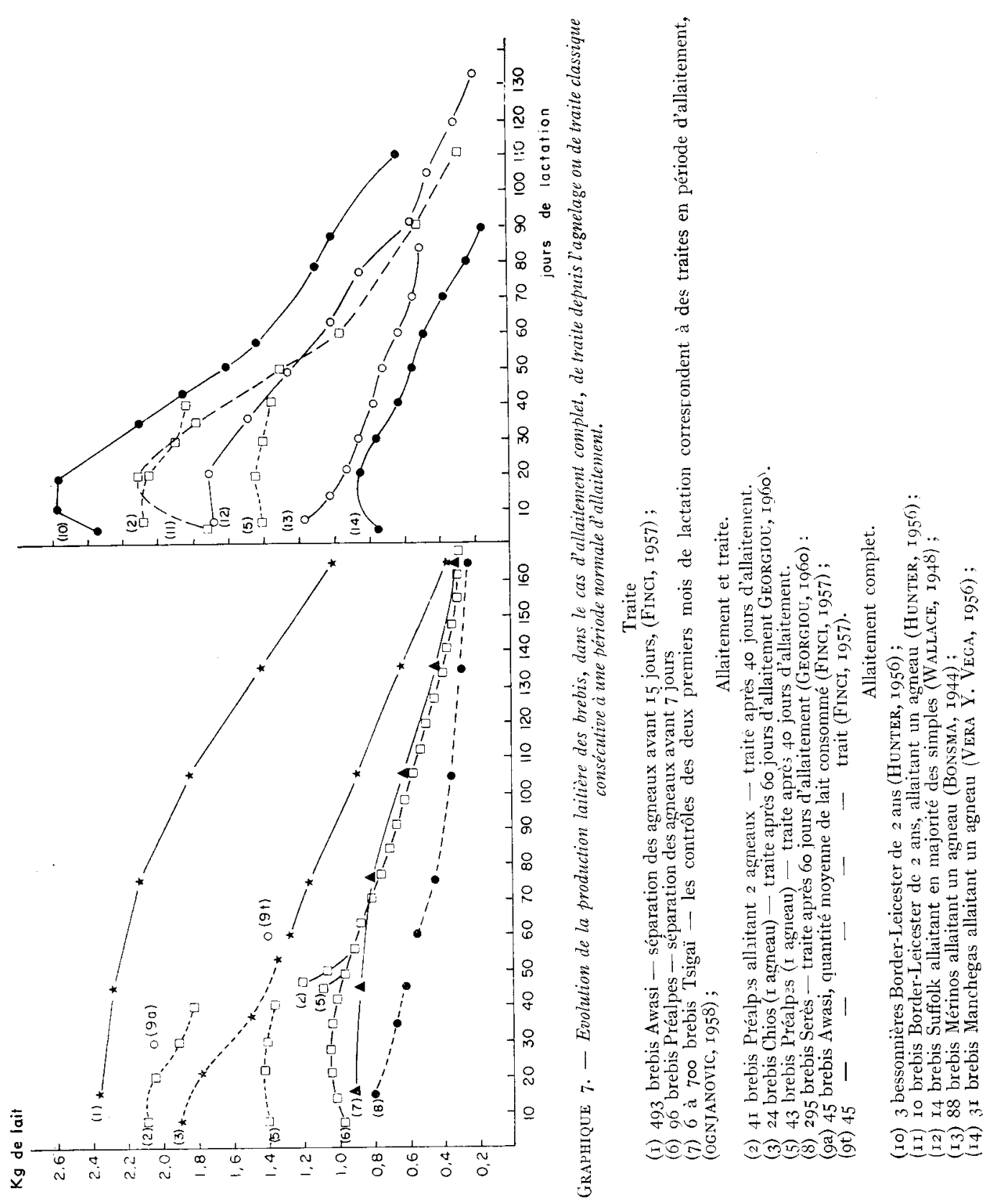


contre, soit en corrélation légèrement négative, soit indépendant de la quantité de lait trait et du volume de la repasse. D'autre part, l'importance du lait consommé par les agneaux dépend trop largement de leur poids et de leur appétit pour que l'on puisse lui attribuer une grande signification.

Da point de vue méthodologie du contrôle laitier, il y a donc intérêt à ne pas intégrer les performances obtenues à la tétée dans le calcul de la lactation totale et de retenir uniquement les performances de traite, en essayant de contrôler ces dernières aussi rapidement que possible après la mise bas.

$5^{\circ}$ Il apparaît finalement, d'après les comparaisons que nous avons multipliées, que les performances de traite traduisent non pas les potentialités laitières de la brebis mais son aptitude à la traite. C'est là un point capital du point de vue sélection.

Les brebis Préalpes ont un comportement maternel très développé et sont capables de nourrir largement leurs agneaux. Cependant, dès que la traite est substituée à l'allaitement, leurs productions laitières s'établissent à un niveau très inférieur, par suite vraisemblablement, d'une insuffisance du réflexe d'éjection. Lors des premiers jours de la mise à la traite mécanique, bien que la glande mammaire soit gonflée de lait, l'écoulement n'intervient pas toujours à la pose des gobelets, mais bien souvent lors de l'égouttage machine. La repasse manuelle consécutive à cet l'égouttage parmat de récupérer une quantité de lait appréciable, mais elle est insuffisante pour réaliser une vidange complète de la mamelle.

Il est certain que la sélection entreprise sur ces souches de brebis, (sélection essentiellement orientée sur la quantité de lait consommé par les agneaux, les éleveurs retenant de préférence pour la reproduction, les animaux les plus vigoureux et les plus précoces) ne pouvait, compte tenu de son efficacité, se traduire par un accroissement notable de l'aptitude laitière des brebis à la traite. Cela est d'autant plus vrai, que certaines races méditerranéennes, excellentes laitières à la traite se révèlent médiocres nourrices. Les chercheurs Israéliens (Eyai, Tolcani, Sharav, I948) vont même jusqu'à prétendre que la brebis laitière idéale ne doit pas avoir un sens maternel très poussé afin de pouvoir être traite facilement.

$6^{\circ}$ Compte tenu de cet ensemble de résultats, comment pouvons-nous concevoir l'amélioration de l'aptitude laitière à la traite des brebis Préalpes? Plusieurs solutions peuvent être envisagées simultanément:

- Sélection er race pu\% grâce à 1'utilisation de reproducteurs jugés améliorateurs. C'est sur la descendance des béliers que doit porter l'essentiel du contrôle des performances, l'intensité de sélection des mâles pouvant être nettement supérieure. à celle des femelles surtout si le testage est organisé avec le concours de l'insémination artificielle.

- Croisement de ces brebis avec d'autres races reconnues faciles à traire. Cette solution doit permettre des progrès rapides, dans la mesure où les produits obtenus répondent aux exigences $d u$ milieu. En fait, actuellement l'amélioration des conditions d'élevage et de production fourragère est telle qu'on peut envisager la création de races peut-être moins rustiques et plus exigeantes, mais nettement plus productives.

- Étude des mécanismes physiologiques de la traile et connaissance de tous les stimuli susceptibles d'améliorer l'écoulement du lait : étude du comportement de la brebis à la traite et en présence de son agneau. 


\author{
SUMMARY \\ MILKING PRODLCTION OF PREALPES RWES DLRING SLCKLIN;, \\ IVEANING ANI MILKING PERIODS
}

'The milk production of P'realpes ewes has been studied during the successive stages of suckling, weaning and milking ( 16 lots and 250 lactations).

I. - The recording of the milk production of ewes milked a few days after lambing gave us the lactation curve of reference. This is characterised by a peried of increasing secretion (during a maximum of 50 days) followed by a feriod of decreasing secretion, the total lactation covering about I 75 days.

The maximum is not very high ( 1,25 litre) and is situated on an average at 25 days (table 3 ). The higher its level is, the earlier it is.

From the 5 th to the 1 joth day of lactation, the rate of weekly decrease of milk production is † p. Ios (table 4).

The milk produced during each of the first 2 months of lactation corresponds to $25 \mathrm{p}$. 100 of the total production obtaincd in 175 days milking.

II. - The phase of increasing production during the suckling period was studied each week, by weighing the lambs before and after suckling.

For ewes with single and with twin lambs, on an average the maximum is situated respectively at 19 and $\mathrm{I} 6$ days and reaches $\mathrm{I}, 7$ and $2,3 \mathrm{~kg}$ (i. e. a difference of $34 \mathrm{p}$. 100, 1able 3 ).

III. - Whatever the method of weaning (brutal weaning or partial suckling - table 8), the number of lambs reared (table 9 ), the length of the suckling period ( 15,30 or 45 days - table ro), the production of milk obtained at milking, after the suckling period, is (with the exception of the 2 first weeks) for the same period of lactation, quite comparable in cluantity and persistency with the production of ewes milked straight after lantbing.

IV. - The comparison of the performances recorded with milking and with suckling, between the end of suckling and the beginning of milking in the case of brutal weaning, between successive periods of milking and suckling in the case of partial suckling, shows that only 60 to 80 p. Ico of the milk normally suckled by the lamb is obtained by milking (table i r). The difference between the quantity of milk suckled and that obtained by milking presented a high positive correlation $\mathrm{w}$ ith the Ist of these variables and a slightly negative correlation with the 2nd; it de ends on the we ght of the lambs, except when they are sufficiently strong to consune all the milk their mcther is able to produce.

There exists in fact, a positive relation between the quantity of milk obtained ty milking and that consumed by the lambs, but this latter has only a relative value and cannot be included without correction in the calculation of the total lactation.

To be effective, it appears clearly that the selection of ewes bred for milk production inust relies essentially on the performances obtained at milking.

V. - The adoption of partial weaning does not eliminate the retention of milk observed after complete ueming of the lambs but it makes possible to begin milki ing a little ear i:r (the milk from partial milking was however poorer in fat -.. table $\mathrm{I}_{3}$ ) and especially $\mathrm{t}, \mathrm{decrease}$ the risks of mastitis particularly in ewes suckling twins.

Immediately after milking in the course of partial suckling, the lambs able to recuperate a quantity of milk, representing $3 . \mathrm{p}$. 1 co of the volume milked. By replacing the suckling of the lamb by milking after an injection of I LI of ocytocine, $62 \mathrm{p}$. roo of this same volume is obtained (table $15)$.

The presence of the lamb at milking during partial weaning does not ensure a bet ter emptying of the udder (table i4).

\title{
RÉFÉRENCES BIBLIOGRAPHIQUES
}

Alexander G., Davies H. I.., 1959. Relationship of milk production to number of lambs born or suckled. Austr. J.agric. Res., 5, 7 20-724.

Barnicoat C. R., Logan A., G., Grant A. J., I949. Milk secretion studies with New-Zealand Romney ewes. Part. I. J. agric. Sci., 39, 44-45.

Barnicoat C. R., Murray P. F., Roberts E. M., Wilsox G. S., 1956. Milk secretion studies with NewZealand Romney ewes. J. agric. Sci., 48, 9-35. 
Betrini T. M., i952. Su alcune cause di variazone della lunghezza della lattazione e della produzione lattea nella pecora Sarda. Riv. Zoolec., 5, i16-123.

Bonelli P., I955. Coefficienti di normalizzazione della produzione lattea pecora sarda. Riv. Zootec., 28, $293-296$.

Bonsma F. N., 1939. Factors influencing the growth and development of lambs with special reference to cross-breeding of Merino-sheep for fat lamb production. Univ. Preloria Publ. Ser. I, Agric. $\mathrm{n}^{\circ} 48$.

Bonsma F. N., I944. Milk production studies with sheep. Iimg in S. Afr., 19, $3_{\text {II-324. }}$

I3osc J., (Communication personnell').

BRUCE H. .I., 1965. Observations on the suckling stimulus and lactation in the rat. J. Reprod. Fertil., 2, r 7.

BroTkAMP., I952. lmang, Leistungen und Fruchtbarkeitverhälnise der ostfrieseschenn Milchschafzucht. Tier $\approx \ddot{u ̈ c h}$ ier., 4, 535 .

Ciolca N., Pirvulescu S., Tafta V., Georgescu D., ig6o. Amélioration de la méthode d'estimation de

la production laitière des Brebis pendant la période d'allaitement. Lucr. Sti. Inst. Cerc. Zootech. (Bucaresti), 18, $369-38 \mathrm{r}$.

Constantinescu ()., Gondos (i., I958. Contrôle de la prodution laitière des Brebis durant la période d'allaitement. Ann. Inst. Cerc. Zootech., 15, $459^{-474}$.

Coombe J. B., WArinrop I. D., Tribe D. E., 1960. A study of milk production of the grazing ewe, with emphasis on the expérimental technique employed. J. agric. Sci., 54, 353-359.

CREMER E., I935. Milchleistungsprüfungen und ihr Ergebnisbeim ostfriesischen Milchshaf. Z. Schafs, 24, I 74-I8O, 203-206.

Davies H. L., 1958. Milk yield of Australian Merino Ewes and lamb growth under pastoral conditions. Proc. Alust. Soc. Anim. Produc., 2, I 5-21.

Jiral E., Volcaxi R., Sharav E., 1958. Milk secretion on problems of milking elficiency in sheep. Hanoked., $\mathbf{4 0 .}$

Eninginaus II., 1940. Der Milchshatë̈hter. Metta Kinat Verlag Nachf. Lunebourg.

Fiver M., 1957. The improvement of the Awasi breed of sheep in [sraël. Bull. Res. Counc. of Israel., 6 B, i- 106.

Georgiou A., ig6o. Sheep and Goat raising in Greece. Congrès FAO, FEZ d' Ahènes, iq6o.

Guyer P. Q., Dyer A. J., I954. Study of factors affecting sheep production. .14issouri Agric. Exp. Stat. Res. Bull., $\mathbf{5 5 8 .}$

Hunter G. G., 1956. The maternal infuence on size in shecp. J. agric. Sci., 48, 36-60.

Kirsch W., I943. Mutton Merino sheep for milk production. In Anim. Breed Absir., 12, 140.

Leroy A. M., I93I. Nouvelles méthode pour le contrôle de l'alimentation des vachés laitières. C.R. Acad. Agric., 28, 937 .

Leroy A. M., 1934. Téthode de contrôle de l'alimentation des vaches laitières. Le lail, 48, 266-274, 366-373.

Leroy A. M., i 936. Application éventuelle des méthodes de contrôle laitier à la sélection des races ovines précoces. C. R. Acad. Agric., 22, 733-738.

Mason I. L., Dassat P., I954. Milk meat and wool production in the Langhe sheep of Italy. Z. Tierz. Ziicht Biol, 62, 197.

Mrreu S., I957. Effetti dell'eta al primo parto, "precone " e "tardivo "sulli produzione lattea, sulla lunghezza della vita produtiva e su altre caratteristiche nella pecora sarda dell'olive di Monastir. Ri Zootec., 30, $54-58$,

OCNJANOvic A., 1958. The influence of twinning and consecutive lactations on milk yield and milk composition of Cigaja sheep. Unir. Beogradu.

Oknotina D. N., Milk production of Askanian cwss. In Dairy Sci, Abstr, 21, 2łr.

OWEN J. B., I955. Milk production in sheep. Agricullure Long., 62, r I0-I 14.

Oven J. B., 1957. A study of the lactation and rrowth of IIill sheep in their native environment and under lowland conditions. J. agric. Sci., 48, 387-+1 2.

Pospelov V. P., 1940. The milk yield of sheep after weaning the lambs in the conditions of South Kazakstan. In. Anim. Breed. Absir., 12, 1944.

Plommet M., Ricordeau G., ig6o. Mammite staphylococcique de la Brebis. Influence des modes de traite et de sevrage, du nombre d'agneaux, du stade de lactation et de la production laitière sur le déclenchement de l'infection. Ann. Zootech., 9, $225-240$.

Ricordeau G., Boccard R., Denamur R., igho. Mesure de la production laitière des Brebis pendant la période d'allaitement. Ann. Zootech., 9, $97^{-1} 20$.

Ricorieau G., Denamur R., ig62. Mesure de la production laitiere des Brebis pendant la période d'allaitement (II). Ann. Zootech, (à paraître).

Ricordeau G., Denamur R,, Martinet J., i962. Observations sur la traite des Brebis Préalpes du Sud. Ann. Zootech. (à paraître).

Scinemacker F., 1929. Cité par ("lrich A., 1953. Die Milch und Sä̈geleistung von Leinesschafen. Zuchtungskunde., 24, $13 \mathrm{I}$.

Schemgraber M., 1934. Der Wert des Schafes als Milchtier unter besonderer Berucksichtigung der verschiedenen Milchschafrassen und ihrer Leistungen. \%. 'Tierz. Zücht I3iol., 28, 103. 
Starke J. S., I953. Studies on the inheritance of milk production in sheep. S. Afr. J. Sci., 49, 245-254. Ternovenko N. M., 1958. Milk yield of ewes of the Caucasian breed. In Dairy Sci. Abstr., 21, 24I.

Vera Y., Vega A., 1956. Comparacion de dos metodos de estimacion de la produccion lechera en la oveja durante el periodo de amamantamiento del cordero. Arch. Zootech., 5, 322-348.

WALLACE L. R., 1948. Growth of lambs before and after birth in relation to the level of nutrition. Part. I. J. agric. Sci., 38, 93-153. 